\title{
Concerto: A Framework for Combined Concrete and Abstract Interpretation
}

\author{
JOHN TOMAN, University of Washington, USA \\ DAN GROSSMAN, University of Washington, USA
}

\begin{abstract}
However, modern software engineering practices pose significant challenges to this vision, specifically the extensive use of frameworks and complex libraries. Frameworks heavily use reflection, metaprogramming, and multiple layers of abstraction, all of which confound even state-of-the-art abstract interpreters. Sound but conservative analysis of frameworks is impractically imprecise, and unsoundly ignoring reflection and metaprogramming is untenable given the prevalence of these features. Manually modeling framework behaviors offers excellent precision, at the cost of immense effort by the tool designer.

To overcome the above difficulties, we present CONCERTO, a system for analyzing framework-based applications by soundly combining concrete and abstract interpretation. CONCERTO analyzes framework implementations using concrete interpretation, and application code using abstract interpretation. This technique is possible in practice as framework implementations typically follow a single path of execution when provided a concrete, application-specific configuration file which is often available at analysis time. ConcerTo exploits this configuration information to precisely resolve reflection and other metaprogramming idioms during concrete execution. In contrast, application code may have infinitely many paths of execution, so CONCERTO switches to abstract interpretation to analyze application code. CONCERTO is an analysis framework, and can be instantiated with any abstract interpretation that satisfies a small set of preconditions. In addition, unlike manual modeling, CONCERTO is not specialized to any specific framework implementation. We have formalized our approach and proved several important properties including soundness and termination. In addition, we have implemented an initial proof of concept prototype of CONCERTo for a subset of Java, and found that our combined interpretation significantly improves analysis precision and performance.
\end{abstract}

\section{CCS Concepts: • Software and its engineering $\rightarrow$ Automated static analysis; Frameworks;}

Additional Key Words and Phrases: abstract interpretation, state separation, reflection, framework-based applications, metaprogramming

\section{ACM Reference Format:}

John Toman and Dan Grossman. 2019. Concerto: A Framework for Combined Concrete and Abstract Interpretation. Proc. ACM Program. Lang. 3, POPL, Article 43 (January 2019), 29 pages. https://doi.org/10.1145/ 3290356

\section{INTRODUCTION}

Modern applications are no longer batch jobs that use only simple data structures and a small set of standard libraries. Instead, to improve productivity and portability, software engineers increasingly rely on large, complex libraries and frameworks to provide the scaffolding on which an application is built. To maximize flexibility, these frameworks are highly configurable and often use

Authors' addresses: John Toman, University of Washington, USA, jtoman@cs.washington.edu; Dan Grossman, University of Washington, USA, djg@cs.washington.edu.

This work is licensed under a Creative Commons Attribution 4.0 International License.

(C) 2019 Copyright held by the owner/author(s).

2475-1421/2019/1-ART43

https://doi.org/10.1145/3290356

Proc. ACM Program. Lang., Vol. 3, No. POPL, Article 43. Publication date: January 2019. 


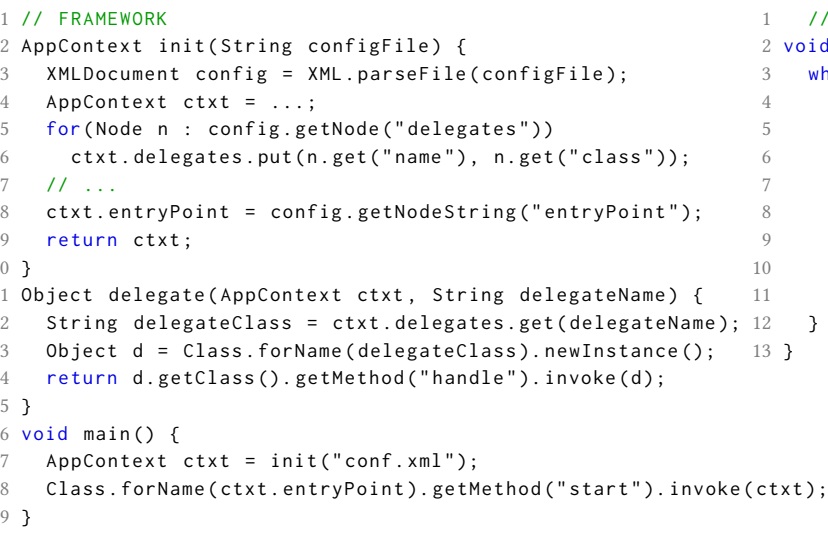

Fig. 1. A motivating example demonstrating the difficulty in analyzing framework applications. Specifically, key control-flow decisions in delegate and main depend on the contents of conf.xml.

metaprogramming, reflection, embedded DSLs, etc. This increased flexibility comes at the cost of precision and/or soundness when using abstract interpretation or other static analysis techniques to reason about the behavior of framework-based applications.

For example, consider the invented code fragment in Fig. 1, which exemplifies code patterns commonly found in frameworks. Here start is part of the application, whereas delegate, init, and main are provided by the framework. The framework first calls init, which parses an applicationspecific XML configuration file, then constructs an Appcontext to hold the framework state. This state consists of a delegates map, which maps names found in the configuration file to corresponding class names, including the application's entry point. The framework invokes the application's entry point on line 18. When the application calls delegate from start, the framework uses delegates to instantiate the class associated with the delegateName argument and then reflectively invokes the handle() method on the newly constructed object. Thus, the callees on lines 14 and 18 are determined entirely by the configuration file, which is opaque to standard call-graph construction algorithms.

To analyze delegate and the reflective method invocation on line 18 , analysis authors can choose to: 1) unsoundly ignore the reflective call, 2) be extremely imprecise, e.g., by allowing reflective calls to resolve to any method, or 3) based on the contents of conf.xml, build an application-specific model of the framework behavior. Option 1 misses most of the application's behavior, yielding many false negatives; in Fig. 1, none of the application will be analyzed. Option 2 has the opposite problem: many false positives and infeasible control-flow paths. Finally, option 3 requires significant manual effort. Although this effort can be alleviated with framework-specific model generators [Sridharan et al. 2011], creating a model generator for a framework is itself a monumental task.

Other analysis techniques also struggle with framework-based applications. Given a concrete configuration file, the framework methods in Fig. 1 follow only one execution path, suggesting a partially-concrete approach, such as concolic execution [Godefroid et al. 2005; Sen and Agha 2006; Sen et al. 2005]. However, the infinite "accept" loop in start is challenging even for state-ofthe-art concolic executors. Finitization of the loop can yield false negatives, and some execution engines may fail to terminate. In contrast, a static analysis built using the monotone framework [Kam and Ullman 1977] or abstract interpretation [Cousot and Cousot 1977, 1979b, 1992b] can soundly approximate the infinite loop by computing a least fixed point over a series of equations.

A key insight of our approach is that many applications and frameworks follow this pattern: framework implementations are difficult to analyze statically, but large parts are statically executable given 
a concrete, application-specific configuration file. Statically executable refers to a program fragment that: a) can be completely and deterministically evaluated at analysis time, and b) will yield the same program state after evaluation at both runtime and analysis time; the init method is an example of statically executable code. Conversely, application code contains unbounded control-flow paths. As a result, a one-size-fits-all approach to program analysis is unwise for framework-based applications.

We present CONCERTO, a system for soundly combining mostly-concrete interpretation, an extension to concrete interpretation we introduce and formalize in this paper, and abstract interpretation. By combining these two techniques, CONCERTo leverages the strengths of both approaches while avoiding their weaknesses. CONCERTO analyzes framework implementations using mostly-concrete interpretation, and application code using abstract interpretation. Mostly-concrete interpretation supports nondeterminism and over-approximation of sets of values, ensuring our combined interpretation is sound while still terminating.

Our combined interpreter is itself an abstract interpreter that operates over a combined domain of abstract and mostly-concrete states. By formalizing our combined approach within the theory of abstract interpretation (AI) we can directly use techniques from the AI literature to prove soundness, termination, etc. CONCERTO differs from partial evaluation, as the abstract and concrete interpreters may yield into one another on demand, which allows greater concrete execution within framework code (we illustrate this point further in Section 2). ConcerTo is analysis agnostic and can be used with any analysis that satisfies a modest set of conditions. It is provably sound: integrating any sound abstract interpretation that satisfies these conditions into CONCERTO yields a sound, combined analysis. In addition, we have shown that abstract interpretations that satisfy a small, additional set of conditions can provably expect equal or greater precision when used with CONCERTO.

Key to our approach is the observation that framework code does not directly manipulate application state and vice versa. This state separation allows CONCERTO to partition a program state into two disjoint representations: a mostly-concrete representation used to model the framework state, and an abstract representation for application state. The mostly-concrete component of CoNCERTO may therefore manipulate its portion of the program state while remaining agnostic about the abstract representation used by the abstract interpretation component, and vice versa.

We have implemented an initial proof of concept of CONCERTO for a subset of Java. We have demonstrated the flexibility of CONCERTO by incorporating three different analyses with different abstract domains into this prototype. We found that using CONCERTO significantly increased precision across all analyses when applied to a difficult-to-analyze framework implementation.

\section{OVERVIEW}

We will first informally describe how CONCERTO operates on the program in Fig. 2, which is written in a simple language we will call MAP. Among other features, MAP supports I/O, reflection, maps which are sufficient to illustrate the core of our technique. This program and language will also serve as our running example as we formalize our approach in the remainder of this paper. We will later formalize a language parameterized by base constants and operations into which we can embed the MAP language.

In Fig. 2 the framework code is in the left column and the application code in the right. On lines 2-9, the framework opens an (application-provided) configuration file, and creates a map $\mathrm{m}$ from application-specific identifiers to procedure names. The application start point, $\mathrm{s}$, is then called with $m$ as its argument. The only other framework code is dispatch, which uses the map produced in main to look up a procedure name associated with $\mathrm{k}$ and invoke the named method. The invoke intrinsic calls the procedure named by the first argument with the remaining arguments. The application-specific logic is implemented in the procedures $s, f, g, h$, and $i$. 


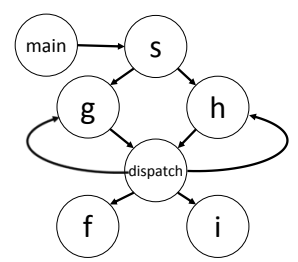

(a) Sound but Imprecise Call-Graph

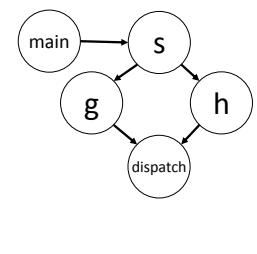

(b) Unsound Call-Graph

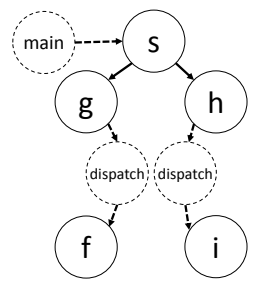

(c) Call-Graph with Concerto

Fig. 3. Call graphs produced by different analysis schemes. In (a), calls from dispatch to main and s can be ruled out by matching argument arities. In (c), procedures executed (mostly-)concretely are given a dashed outline.

defined interfaces to communicate with the application. Together, these facts suggest that modern Java framework implementations are a natural fit for our state separation hypothesis.

CONCERTO exploits this state separation to thread abstract values produced by the abstract interpreter through concrete interpretation and the abstract interpretation may do the same for concrete values produced by concrete interpretation. (CONCERTO also includes support for the rare cases where this hypothesis does not apply, see Section 8.4.) Section 3.1 formalizes a type-based state separation that is natural in languages like Java.

Without additional knowledge about the program in Fig. 2, a standard abstract interpretation not integrated with CONCERTO must make worst-case assumptions about read, and thus use an extremely imprecise abstraction of the framework state in $\mathrm{m}$. As a result, analysis of dispatch would conclude that invoke may call any procedure. Thus, plain abstract interpretation cannot rule out that a negative argument may flow from $h$ through dispatch to $f$ and that the error() statement is reachable. On the other hand, ignoring invoke as though it is a no-op ignores important application behavior. These two situations are illustrated in Figs. 3a and 3b, respectively.

Suppose now that we have the following domain knowledge about our program:

(1) The contents of the file config are available at analysis time and will not change between analysis time and program runtime.

(2) config has the contents shown in Fig. 2

This information ensures that error() is never executed: dispatch will always call $f$ with the positive argument passed to it by g. However, even if an abstract interpretation has this information, verifying that error() is unreachable requires an extremely precise semantics and representation for maps. This can be achieved in this simple language, but, in practice, frameworks use much more complicated data structures and abstractions, making precise analysis via pure abstract interpretation unlikely. In contrast, CONCERTO integrated with a simple signedness analysis can prove that the error() statement is unreachable, using a process we briefly sketch below.

\subsection{Analyzing the Example}

CONCERTo begins analysis of the program by concretely executing main(). Due to the domain-specific knowledge described above, the initialization loop is statically executable. Thus, Concerto opens the file "config" and runs the loop to completion. When the loop terminates, m holds the map ["b" $\mapsto$ "f", "a" $\mapsto$ "i"]. We stress that ConcERTo uses no application- or analysis-specific logic here: CONCERTo simply performs concrete interpretation, opening the listed file and executing the loop.

At the call to the application entry point s on line 11, CONCERTO switches to abstract interpretation, in this example a signedness analysis. A key assumption of CONCERTO is that framework 
code, once given a concrete configuration, is almost entirely statically executable. In contrast, application code may deal with nondeterministic inputs, giving rise to unbounded loops like the one on lines 23-26. Although concrete execution will naturally fail to terminate on the loop in s, abstract interpretation can easily over-approximate the loop.

When switching to abstract interpretation, CONCERTO transforms the concrete program state at the call-site to the abstract representation used by the abstract interpreter. We describe this process in more detail in Section 4.3. In this example, the signedness analysis begins in the abstract state: $[m \mapsto[" b " \mapsto " f ", " a " \mapsto " i "]]$. The abstract interpreter has not abstracted away the framework state; instead, the signedness analysis has reused the concrete value directly for the value of $m$. However, the abstract interpreter does not need to implement concrete map semantics or otherwise "understand" this representation. Due to the state separation hypothesis described above, any map operations on $\mathrm{m}$ occur in framework code, which is not analyzed using abstract interpretation

In the while loop of $\mathrm{s}$, the signedness analysis analyzes the call to g, which itself calls the framework's dispatch procedure in the abstract state $[m \mapsto[" b " \mapsto " f ", " a " \mapsto " i "], p \mapsto\{+\}]$. For this call, CONCERTO switches from abstract interpretation to mostly-concrete interpretation. CONCERTO cannot switch back to fully-concrete interpretation soundly because the above abstract state cannot be concretized to a single concrete state (or even a finite set of states): $p$ abstracts the infinite set of all positive integers. To avoid materializing an infinite set of concrete states, our mostlyconcrete interpretation supports runtime values that are abstractions of infinite sets of values. To represent the infinite set of possible values of $p$, the mostly-concrete interpreter reuses p's abstract value in the abstract interpreter, i.e., $\{+\}$. CONCERTO is agnostic to the domain of abstract values; had the analysis chosen instead to represent integers with, say, intervals, the mostly-concrete interpreter would also use intervals. As with the embedding of concrete maps into abstract states, the mostlyconcrete interpreter does not need semantics over the signedness domain: integer operations on the application state only occur in application code which is not executed mostly-concretely.

As the value of $m$ in the abstract caller state is ["b" $\mapsto " f ", " a " \mapsto " i "]$, this value can be directly reused in the callee mostly-concrete state. Hence, mostly-concrete interpretation has no problem concretely evaluating this call to dispatch with "b" and $\mathrm{m}$ to determine that control should transfer back to the application by calling $f$ with $p$.

At this final call back into the application, CONCERTO once again switches to abstract interpretation, transforming the mostly-concrete state into an abstract state. The value of arg in the mostlyconcrete state is $\{+\}$, which becomes the value of $p$ in the abstract interpreter. Using this abstract value, the abstract interpreter can prove the true branch of the conditional is never taken. Analysis of the call to $\mathrm{h}$ on line 27 proceeds similarly, again using mostly-concrete interpretation to precisely resolve the dispatch call. The final call-graph used during combined interpretation is shown in Fig. 3c.

The above process bears many similarities to partial evaluation [Futamura 1999; Mogensen 1995] where the configuration file is treated as a static input. A sufficiently powerful partial evaluator that supports metaprogramming (e.g., [Sullivan 2001]), could produce a residual program from Fig. 2 that a signedness analysis could verify. However, suppose g used dynamic input to choose between "b" and another procedure name as the argument to dispatch. In this scenario, the partial evaluator would not fully reduce the body of dispatch, making the signedness analysis imprecise. In contrast, under CONCERTO, provided the abstract interpretation faithfully tracks the two possible procedure names, the mostly-concrete interpreter can precisely resolve the invoke operation to the two potential callees.

\subsection{Paper Outline}

The remainder of this paper formalizes and elaborates on the process sketched above. Section 3 defines an intraprocedural language we will use and extend throughout this paper. Our formal

Proc. ACM Program. Lang., Vol. 3, No. POPL, Article 43. Publication date: January 2019. 


\begin{tabular}{|c|c|c|c|c|c|}
\hline & $::=$ & $\ell_{a} \mid \ell_{f}$ & prog & $::=$ & $\left(f_{s t m t} t^{\ell} \mid \operatorname{astmt}^{\ell}\right)^{*}$ \\
\hline astmt $^{\ell}$ & $::=$ & $\ell_{a}:$ astmt & fstmt $^{\ell}$ & $::=$ & $\ell_{f}: f_{s t m t}$ \\
\hline $\begin{array}{r}\text { astmt } \\
\text { aexp }\end{array}$ & $\begin{array}{l}::= \\
::=\end{array}$ & $\begin{array}{l}\text { goto } \ell|x=\operatorname{aexp}| \text { if } x<=>y \text { goto } \ell \\
y\left|b c_{a}\right| \operatorname{aop}\left(v_{1}, \ldots, v_{n}\right)\end{array}$ & $\begin{array}{l}\text { fstmt } \\
\text { fexpr }\end{array}$ & $\begin{array}{l}::= \\
::=\end{array}$ & $\begin{array}{l}\text { goto } \ell|x=f \exp | \text { if } x<=>y \text { goto } \ell \\
x\left|b c_{f}\right| \text { fop }\left(v_{1}, \ldots, v_{n}\right)\end{array}$ \\
\hline
\end{tabular}

Fig. 4. Intraprocedural grammar, parameterized by language-specific choices for $b c_{f}$, fop, $b c_{a}$, and $a o p$.

language makes explicit the state separation hypothesis. We define a concrete semantics for this language against which we prove CONCERTO sound, and describe the expected definition of abstract interpreters.

Section 4 describes our main contribution: the combination of concrete and abstract interpretation. Section 4.1 demonstrates how a naïve combination of concrete and abstract interpretation yields a sound basis for combined interpretation, but one that is impractical due to the difficulty of concretizing abstractions of infinite sets of values, as was the case with $\{+\}$ above. Section 4.2 defines mostly-concrete interpretation which can handle abstractions of infinite sets of values. Section 4.3 shows how combining abstract and mostly-concrete interpretation yields a sound interpretation, and formalizes how mostly-concrete states are transformed into abstract states, and vice versa. Finally, Section 4.4 defines a set of sufficient conditions for CONCERTO to match or exceed the precision of an abstract interpretation.

Section 5 briefly discusses how we extend our formalism to procedures. Section 6 describes a particular iteration strategy that is natural in practice and Section 7 sketches how we ensure termination (while retaining soundness) under this iteration strategy using widening. We then discuss our prototype implementation (Section 8), and the results of initial case studies (Section 9). We close with a discussion of related work (Section 10) and future work (Section 11).

\section{PRELIMINARY DEFINITIONS}

\subsection{Language Definition}

Our core calculus is a simple imperative language with conditional/unconditional goto, variable assignments, and constants and primitive operations over a set of types. We formalize a typebased state separation by partitioning this set of types into application and framework types, and restricting all operations on framework types to framework code and similarly for application types.

The formal grammar is given in Fig. 4. We assume two disjoint families of types $\mathcal{A}$ (for application) and $\mathcal{F}$ (for framework) and, with a slight abuse of notation, will use $\mathcal{A}$ (respectively $\mathcal{F}$ ) as a metavariable to range over the types in $\mathcal{A}$ (respectively $\mathcal{F}$ ). Every variable is given a type drawn from one of these families. $b c_{f}$ and $f o p$ range over base constants and primitive operations respectively for types in $\mathcal{F}$, and $b c_{a}$ and $a o p$ do the same for $\mathcal{A}$. All operations in fop have type $(\mathcal{F} \times$ $\ldots \times \mathcal{F}) \rightarrow \mathcal{F}$, and similarly for aop and $\mathcal{A}$. The $\Leftrightarrow=>$ nonterminal ranges over comparison operators.

In our language, $\ell_{f}$ label framework statements, and similarly for $\ell_{a}$ and application statements. The label of a statement determines what operations may be performed by that statement: fops and $b c_{f}$ may only appear in code labeled with $\ell_{f}$, and similarly for $a o p, b c_{a}$ and $\ell_{a}$. Further, we require that comparisons in statements labeled with $\ell_{a}$ can only compare values of types in $\mathcal{A}$, and similarly for $\ell_{f}$ and $\mathcal{F}$. However, statements labeled $\ell_{f}$ may move values between variables with type $\mathcal{A}$, and vice versa for statements labeled $\ell_{a}$. As such, framework code must treat values of type $\mathcal{A}$ ("application values") opaquely, threading them through to statements labeled $\ell_{f}$. Similarly, statements labeled $\ell_{a}$ treat $\mathcal{F}$ values opaquely. This syntactic restriction models the state separation hypothesis described in Section 2. (In practice, this strict separation may be violated by, e.g., primitive types like int, library types, etc.; our implementation has special support for these shared types as described in Section 8.4.) 
The full operational semantics (omitted for space reasons) are defined in terms of the following denotations and value domains, which we will use throughout the remainder of the paper. Let $V_{a}$ be the set of all values with a type in $\mathcal{A}$ and similarly for $V_{f}$ and $\mathcal{F}$. Every aop has a denotation $\llbracket a o p \rrbracket:\left(V_{a} \times \ldots V_{a}\right) \rightarrow V_{a}$; we assume that aops are deterministic. In contrast, some fop operations may produce values that depend on the value of some environment model $\mathcal{E}$, which models nondeterminism due to file contents, network requests, etc. Formally, each fop has a denotation $\llbracket f o p \rrbracket: \mathcal{E} \times V_{f} \times \ldots \times V_{f} \rightarrow \wp\left(V_{f} \times \mathcal{E}\right)$, where $\left\langle v^{\prime}, E^{\prime}\right\rangle \in \llbracket f o p \rrbracket\left(E, v_{1}, \ldots, v_{n}\right)$ means executing fop in environment $E \in \mathcal{E}$ with arguments $v_{1}, \ldots, v_{n}$ produces a new environment model $E^{\prime}$ and result $v^{\prime}$. To simplify presentation, we require that denotations are total functions; in practice, we assume that the denotations gracefully handle runtime type errors (e.g., by returning a sentinel error value, halting execution, etc.). Finally, we assume that $\llbracket<=>\rrbracket$ denotes into a binary relation over values of the appropriate type, $\llbracket\langle\neq\rangle \rrbracket$ is the negation of $\llbracket<=>\rrbracket$, and $\llbracket b c_{f} \rrbracket$ and $\llbracket b c_{a} \rrbracket$ produce values in $V_{f}$ and $V_{a}$ respectively corresponding to the interpretation of those constants.

Although the result of fops depend on the current environment $E$, in some cases we may have a priori information such that a seemingly nondeterministic fop, i.e., reading a file, is effectively deterministic. For example, in Section 2, we exploited domain specific information about runtime contents of the configuration file to precisely execute the initialization loop. We account for this knowledge by allowing hypotheses on the domain of $\mathcal{E}$. For example, if $\mathcal{E}$ models file-system contents, and we have a priori knowledge that a file $f$ always has content $c$ at runtime, we can restrict $\mathcal{E}$ to include only models where the file $f$ has contents $c$.

Throughout the rest of this paper we assume that we are operating on some arbitrary program written in this language and that the relations pred and succ are defined with the obvious definitions and there is a map prog from labels to unlabeled statements.

Example 3.1 (MAP Language). We can encode the MAP language and state separation of Section 2 in this language framework as follows. Control-flow constructs (if/while) can be encoded using the goto representation defined in Fig. 4; we defer discussion of procedures to Section 5.

We take $\mathcal{A}=\{$ int $\}$, i.e., the type of machine integers, with $b c_{a}$ defined to be integer constants, aop to be the usual arithmetic operations, and $V_{a}$ as machine integers. We next define $\mathcal{F}=\{$ File, Str, $\mathcal{M}\}$, where $\mathcal{M}$ is the domain of maps from strings to strings, and instantiate fop with the following:

$$
\text { open : Str } \rightarrow \text { File } \quad \text { read : File } \rightarrow \text { Str } \quad \text { set }: \mathcal{M} \times S t r \times S t r \rightarrow S t r \quad \text { get }: \mathcal{M} \times S t r \rightarrow \text { Str }
$$

We define $b c_{f}$ as the set of literal string constants and empty : $\mathcal{M}$ which is an empty map. Finally, we take $V_{f}=S T R \cup$ FileContents $\cup(S T R \rightarrow S T R)$, where $S T R$ is the set of string values and FileContents is a finite stream of $S T R$ values. Given the syntactic constraints on where aops and fops may appear, this instantiation encodes that the application may not manipulate the framework dispatch map m, nor may the framework manipulate integers received from the application.

Finally, the domain of environment models $\mathcal{E}$ is a map from strings to file contents, i.e., $S T R \rightarrow$ FileContents. We encode the information about the configuration file by requiring that: $\mathcal{E}=\{e \mid$

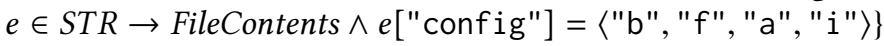

\subsection{Concrete Properties}

CONCERTO targets abstract interpretations where the approximated concrete property is the set of reaching concrete states that may occur during program execution. In the intraprocedural language described thus far, a concrete state is a valuation for the variables in the program plus an environment model. Formally, a concrete state is defined as: $S=\mathcal{E} \times(X \rightarrow V)$ where $V=V_{a} \cup V_{f} \cup\left\{\perp_{V}\right\}, X$ is the domain of variables appearing in a program, $\mathcal{E}$ is type of environment models described in Section 3.1, and $\perp_{V}$ is a sentinel "uninitialized" value. We assume the type system is sound, i.e.,

Proc. ACM Program. Lang., Vol. 3, No. POPL, Article 43. Publication date: January 2019. 


$$
\begin{aligned}
& F(r)\left[\ell^{\circ} \sim \ell^{\bullet}\right]=\bigsqcup_{\substack{p \in \operatorname{pred}(\ell) \\
i n \in r\left[\boldsymbol{p}^{\bullet} \sim \ell^{\circ}\right]}} \operatorname{step}^{F}(\text { in }, \ell) \sqcup \begin{cases}\bigsqcup_{e \in \iota_{\mathcal{E}}} \operatorname{step}^{F}\left(\left\langle\iota_{S}, e\right\rangle, \ell\right) & \ell=s \\
\emptyset & \text { o.w. }\end{cases} \\
& F(r)\left[p^{\bullet} \sim \ell^{\circ}\right]= \begin{cases}\left\{\langle s, E\rangle \mid\langle s, E\rangle \in r\left[p^{\circ} \sim p^{\bullet}\right] \wedge s[x] \llbracket<=>\rrbracket s[y]\right\} & \text { prog }[p]=\text { if } \mathrm{x}<=>\text { y goto } \ell \\
\left.\langle\langle s, E\rangle|\langle s, E\rangle \in r\left[p^{\circ} \sim p^{\bullet}\right] \wedge(s[x] \llbracket<\neq>\rrbracket s[y])\right\} & \text { prog }[p]=\text { if } \mathrm{x}<=>\text { y goto } \ell^{\prime} \\
r\left[p^{\circ} \leadsto p^{\bullet}\right] & \text { o.w. }\end{cases} \\
& \operatorname{step}^{F}(\langle i n, E\rangle, \ell)= \begin{cases}\{\langle\text { in }, E\rangle\} & \operatorname{prog}[\ell]=\text { if ... } \vee \text { goto ... } \\
\{\langle\text { in }[x \mapsto i n[y]], E\rangle\} & \operatorname{prog}[\ell]=x=y \\
\{\langle i n[x \mapsto \llbracket c \rrbracket, E\rangle\} & \operatorname{prog}[\ell]=x=c \\
\left\{\left\langle\text { in }\left[x \mapsto \llbracket a o p \rrbracket\left(i n\left[v_{1}\right], \ldots, \operatorname{in}\left[v_{n}\right]\right)\right], E\right\rangle\right\} & \operatorname{prog}[\ell]=x=\operatorname{aop}\left(v_{1}, \ldots, v_{n}\right) \\
\left\{\left\langle i n[x \mapsto r], E^{\prime}\right\rangle \mid\left\langle r, E^{\prime}\right\rangle \in \llbracket f o p \rrbracket\left(E, \operatorname{in}\left[v_{1}\right], \ldots, \operatorname{in}\left[v_{n}\right]\right)\right\} & \operatorname{prog}[\ell]=x=f o p\left(v_{1}, \ldots, v_{n}\right)\end{cases}
\end{aligned}
$$

Fig. 5. Concrete semantic function. $c$ is any constant of type $\mathcal{A}$ or $\mathcal{F}$ and $\llbracket c \rrbracket$ is its corresponding denotation.

for any concrete state $s$ arising during execution: type $(x) \in \mathcal{F} \Rightarrow s[x] \in V_{f}$ and type $(x) \in \mathcal{A} \Rightarrow$ $s[x] \in V_{a}$, and that all variables must be defined before use, i.e., a program never observes $\perp_{V}$.

Next, define a domain of flow edges $\mathcal{L}$ as: $\mathcal{L}=\left\{\ell^{\circ} \leadsto \ell^{\bullet}\right\} \cup\left\{p^{\bullet} \leadsto \ell^{\circ} \mid p \in \operatorname{pred}(\ell)\right\}$, where $\ell^{\circ}$ and $\ell^{\bullet}$ respectively denote the "entrance to" and "exit from" $\ell$. Elements $\ell^{\circ} \sim \ell^{\bullet}$ of $\mathcal{L}$ correspond to the flow of program control through the statement labeled $\ell$, whereas an element $p^{\bullet} \leadsto \ell^{\circ}$ corresponds to the flow from some predecessor $p$ to $\ell$. When convenient, we will abbreviate $\ell^{\circ} \leadsto \ell^{\bullet}$ as simply $\ell$. We will use $\vec{\ell}$ to represent an arbitrary element of $\mathcal{L}$.

Our domain of concrete properties is $R=\mathcal{L} \rightarrow \wp(S)$, which forms a complete lattice, defined pointwise over edges; the powerset of states also forms a complete lattice with the usual definitions.

We now define the concrete semantic function, $F: R \rightarrow R$, the least fixed-point of which is a complete set of reaching states for a program. That is, for every $\vec{\ell} \in \mathcal{L}$, if $\left\langle s, E^{\prime}\right\rangle \in(l f p F)[\vec{\ell}]$ then there is some execution that flows through edge $\vec{\ell}$ yielding $\left\langle s, E^{\prime}\right\rangle$. The full definition of $F$ is given in Fig. 5. We assume program execution begins at a single, distinguished label $s$. Program start is modeled with the second term in the definition of $F(r)\left[\ell^{\circ} \leadsto \ell^{\bullet}\right]: \iota_{\mathcal{E}}$ is a set of possible initial environments, and $\iota_{S}$ is a distinguished start state which maps all variables to $\perp_{V}$.

\subsection{Abstract Properties}

CONCERTO is designed to combine mostly-concrete execution with an abstract interpretation defined as follows. We assume that sets of reaching concrete states are over-approximated by the complete lattice $\widehat{S}$ with ordering and least upper bound operator $\sqsubseteq_{\widehat{S}}$ and $\sqcup_{\widehat{S}}$ respectively. (We will use $\sqsubseteq_{D}$ to indicate a partial order on a domain $D$.) The domain of abstract properties, $\widehat{R}=\mathcal{L} \rightarrow \widehat{S}$, also forms a complete lattice defined by the pointwise extension of $\sqsubseteq_{\widehat{S}}$ and $\sqcup_{\widehat{S}}$. We further assume that the domain $\widehat{R}$ forms a Galois connection with the domain of concrete properties $R$, defined by

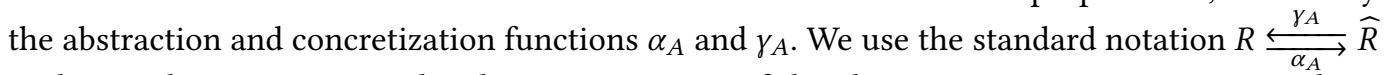
to denote this connection. The abstract semantics of the abstract interpretation are given by a monotone abstract semantic function $\widehat{F}: \widehat{R} \rightarrow \widehat{R}$. We assume that this function is a sound abstraction of $F$ according to the above Galois connection, i.e., $\alpha_{A} \circ F \sqsubseteq_{R \rightarrow \widehat{R}} \widehat{F} \circ \alpha_{A}{ }^{3}$ whence by [Cousot and Cousot 1979b] we have that $\alpha_{A}(l f p F) \sqsubseteq_{\widehat{R}} l f p \widehat{F}$. In other words, the reaching states computed by the least fixed point of $F$ are soundly over-approximated according to $\alpha_{A}$ by the least fixed point of $\widehat{F}$.

Thus, an abstract interpretation is defined by the 7-tuple $\left\langle\widehat{R} ; \widehat{S} ; \widehat{F} ; \alpha_{A} ; \gamma_{A} ; \sqcup_{\widehat{S}} ; \sqsubseteq_{\widehat{S}}\right\rangle$ where:

$$
\widehat{R}=\mathcal{L} \rightarrow \widehat{S} \quad R \underset{\alpha_{A}}{\stackrel{\gamma_{A}}{\leftrightarrows}} \widehat{R} \quad \alpha_{A} \circ F \sqsubseteq_{R \rightarrow \widehat{R}} \widehat{F} \circ \alpha_{A}
$$

$\overline{{ }^{3} \text { Equivalently, }} \alpha_{A} \circ F \circ \gamma_{A} \sqsubseteq_{\widehat{R} \rightarrow \widehat{R}} \widehat{F}$ or $F \circ \gamma_{A} \sqsubseteq_{\widehat{R} \rightarrow R} \gamma_{A} \circ \widehat{F}$. 


$$
\begin{aligned}
& \widehat{F}(\widehat{r})\left[\ell^{\circ} \leadsto \ell^{\bullet}\right]=\operatorname{step}^{+}\left(\bigsqcup_{p \in p \operatorname{pred}(\ell)} \widehat{r}[p], \ell\right) \sqcup \begin{cases}\operatorname{step}^{+}(\perp, \ell) & \ell=s \\
\perp & \text { o.w. }\end{cases} \\
& \widehat{F}(\widehat{r})\left[p^{\bullet} \leadsto \ell^{\circ}\right]= \begin{cases}\widehat{r}\left[p^{\circ} \leadsto p^{\bullet}\right][x \mapsto \operatorname{lop} \cap\{0,+\}] & \text { if } \operatorname{prog}[s]=\text { if } x>=0 \text { goto } \ell \wedge \operatorname{lop} \cap\{0,+\} \neq \emptyset \\
\perp & \text { if } \operatorname{prog}[s]=\text { if } x<=0 \text { goto } \ell^{\prime} \wedge \operatorname{lop} \cap\{-, 0\}=\emptyset \\
\cdots & \text { o.w. }\end{cases}
\end{aligned}
$$

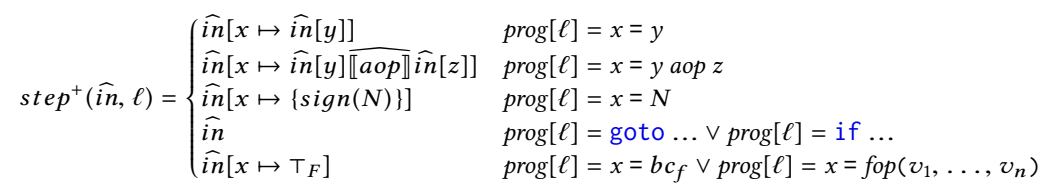

Fig. 6. The abstract semantics for our running signedness example. In the above, we use an infix notation for aop as we assume all integer operations are binary. In the ste $p^{+}$function, $N$ is an integer constant; in the definition of $\widehat{F}$, lop is $\widehat{r}\left[p^{\circ} \leadsto p^{\bullet}\right][x]$ and $s$ is the distinguished start label.

Example 3.2 (Signedness Analysis). The abstract state for the intraprocedural signedness analysis discussed in Section 2 is $\widehat{S}=X_{f} \rightarrow \wp\left(V_{f}\right)^{\top} \times X_{a} \rightarrow \wp(\{-, 0,+\})$, and $\alpha_{A}$ is defined as:

$$
\alpha_{A}(r)[\vec{\ell}]=\left\langle\lambda x: X_{f} \cdot\left\{s[x] \mid\langle s, e\rangle \in r[\vec{\ell}] \wedge s[x] \neq \perp_{V}\right\}, \lambda x: X_{a} \cdot\{\operatorname{sign}(s[x]) \mid\langle s, e\rangle \in r[\vec{\ell}]\}\right\rangle
$$

In the above definitions, $X_{f}$ is the set of program variables with type in $\mathcal{F}, X_{a}$ are those with types in $\mathcal{A}$, and sign returns one of,+- , or 0 depending on the sign of its argument. We omit the definition of $\gamma_{A}$ as it can be derived from the definition of $\alpha_{A}$ [Cousot and Cousot 1979b]. The domain $\wp\left(V_{f}\right)^{\top}$ is the powerset domain of concrete values, extended with a special $\top_{F}$ value, which represents any possible value of type $\mathcal{F}$. We omit the definitions of $\sqsubseteq_{\widehat{S}}$ and $\sqcup_{\widehat{S}}$ as they are standard.

The abstract semantic function $\widehat{F}$ is defined in Fig. 6. We have included only the comparison rules necessary to verify the program in Fig. 2. We omit the definitions for $\widehat{\llbracket+\rrbracket}, \widehat{\llbracket-\rrbracket}$, etc. however $\widehat{\llbracket+\rrbracket}$ is defined such that $\{0,+\} \widehat{\llbracket+\rrbracket}\{+\}=\{+\}$, which again is sufficient to verify the example in Fig. 2.

\section{COMBINED INTERPRETATION}

Given the language, semantic functions, and Galois connection defined in Section 3, we can define an initial, naïe attempt at combined interpretation. Intuitively, this strawman combination, which we call CONCERTO ${ }_{0}$, analyzes framework code by applying the concrete semantic function $F$ at framework statements and applying the abstract semantic function $\widehat{F}$ at application statements. CONCERTO $_{0}$ translates between abstract and concrete states using abstraction and concretization functions. This approach is sound (as proved in the following Section 4.1) but ultimately infeasible to implement as it requires materializing infinite sets of states and values. To overcome this limitation, we extend concrete interpretation to mostly-concrete interpretation. Mostly-concrete interpretation avoids materializing infinite sets by using finite abstractions of sets of possible values. We then define the combination of mostly-concrete and abstract interpretation used by CONCERTO and prove it sound. This combination must translate between different state representations. Unlike CONCERTO , we do not use explicit abstraction or concretization functions. Instead we formalize domain transformers which soundly translate between state domains but are weaker than a Galois connection. We close by proving when CONCERTO matches or exceeds the precision of plain abstract interpretation.

\subsection{Naïve Combination}

To motivate the need for mostly-concrete interpretation, we elaborate on the strawman CONCERTO $_{0}$ and enumerate why it is impractical as a basis for combined analysis. 
CONCERTO $_{0}$ executes framework code (statements with label $\ell_{f}$ ) concretely and abstractly interprets application code (statements with label $\ell_{a}$ ). Our initial attempt at combined interpretation therefore operates over a combined semantic domain that represents reaching states in the framework with sets of concrete states, and reaching states in the application with the abstract state domain $\widehat{S}$. However, the semantic function $F$, which models concrete execution, operates over the fully-concrete domain $R$. Thus, the combined domain is injected into the fully-concrete domain by applying a concretization function to the abstract states of the combined domain. Symmetrically, to abstractly execute application code with the abstract semantic function $\widehat{F}$, the combined domain is injected into the abstract domain by applying an abstraction function to the reaching concrete state component. After injection and applying both semantic functions, $\mathrm{CONCERTO}_{0}$ combines the concrete results at framework statements and abstract results at application statements.

As the combined interpretation uses the highly precise concrete semantics for framework statements (and therefore fops), CONCERTO 0 can, at least in principle, precisely analyze framework code. The concrete interpreter may also use the hypotheses on runtime environments to gain further precision. For example, if the framework parses a configuration file as in the example of Section 2, the concrete interpreter may simply open and parse the configuration file directly. However, not all fops will be analysis-time deterministic, leading to an explosion in reaching concrete states. Further, CONCERTO $_{0}$ relies on an explicit concretization function which cannot be implemented in practice.

We formalize the informal description above as follows. First, we partition the space of $\mathcal{L}$ into two sets, $\mathcal{L}_{A}=\left\{\ell_{a}^{\circ} \leadsto \ell_{a}^{\bullet}\right\} \cup\left\{\ell_{a}^{\bullet} \leadsto \ell^{\circ} \mid \ell_{a} \in \operatorname{pred}(\ell)\right\}$ and $\mathcal{L}_{F}=\left\{\ell_{f}^{\circ} \leadsto \ell_{f}^{\bullet}\right\} \cup\left\{\ell_{f}^{\bullet} \leadsto \ell^{\circ} \mid \ell_{f} \in \operatorname{pred}(\ell)\right\}$. $\mathcal{L}_{A}$ are flow edges originating in $\ell_{a}$-labeled statements, and symmetrically for $\mathcal{L}_{F}$ and $\ell_{f}$. We define this combined domain $\bar{R}_{0}$ and an abstraction function $\alpha_{0}: R \rightarrow \bar{R}_{0}$ as:

$$
\bar{R}_{0}=\mathcal{L}_{F} \rightarrow S \times \mathcal{L}_{A} \rightarrow \widehat{S} \quad \alpha_{0}(r)=\left\langle\lambda \vec{\ell}: \mathcal{L}_{F} \cdot r[\vec{\ell}], \lambda \vec{\ell}: \mathcal{L}_{A} \cdot \alpha_{A}(r)[\vec{\ell}]\right\rangle
$$

$\bar{R}_{0}$ is a complete lattice with the standard component-wise definitions of least upper bound and ordering. As $\alpha_{0}$ is a monotone, complete join morphism there is some $\gamma_{0}$ such that $R$ and $\bar{R}_{0}$ form a Galois connection. We further assume that the abstract and concrete states form a Galois connection $S \underset{\alpha_{S}}{\stackrel{\gamma_{S}}{\leftrightarrows}} \widehat{S}$ and that $\alpha_{A}=\dot{\alpha_{S}}$ and $\gamma_{A}=\dot{\gamma_{S}}$, where $\dot{f}$ denotes the pointwise extension of $f$. Using $\alpha_{S}$ and $\gamma_{S}$, the injection functions described above are defined as:

$$
\operatorname{inj}_{R}(\langle m, \widehat{m}\rangle)[\vec{\ell}]=\left\{\begin{array}{ll}
m[\vec{\ell}] & \vec{\ell} \in \mathcal{L}_{F} \\
\gamma_{S}(\widehat{m}[\vec{\ell}]) & \text { o.w. }
\end{array} \quad \operatorname{inj}_{\widehat{R}}(\langle m, \widehat{m}\rangle)[\vec{\ell}]= \begin{cases}\alpha_{S}(m[\vec{\ell}]) & \vec{\ell} \in \mathcal{L}_{F} \\
\widehat{m}[\vec{\ell}] & \text { o.w. }\end{cases}\right.
$$

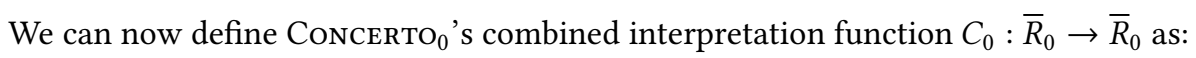

$$
C_{0}(X)=\left\langle\left. F\left(\operatorname{inj}_{R}(X)\right)\right|_{\mathcal{L}_{F}},\left.\widehat{F}\left(\operatorname{inj}_{\widehat{R}}(X)\right)\right|_{\mathcal{L}_{A}}\right\rangle
$$

Theorem 1. $C_{0}$ is sound, i.e., $\alpha_{0} \circ F \sqsubseteq_{R \rightarrow \bar{R}_{0}} C_{0} \circ \alpha_{A}$.

Proof. By the assumed soundness of $\widehat{F}$, that $i n j_{\widehat{R}} \circ \alpha_{0}=\alpha_{A}$ and that $i n j_{R} \circ \alpha_{0}$ is extensive.

Theorem 1 establishes $C_{0}$ is sound, but that doesn't mean $C_{0}$ is a good idea. In fact, it is not. Using $C_{0}$ as the basis for combined interpretation is impractical for the following reasons:

(1) Infinite Sets In many cases, concretizing an abstract state will yield an infinite number of concrete states. For example, concretizing the abstract state $[m \mapsto[" b " \mapsto " f ", " a " \mapsto " i "], p \mapsto$ $\{+\}]$ at the call to dispatch in Section 2 yields the following set of concrete states:

$$
\bigcup_{e \in \mathcal{E}}\{\langle e,[m \mapsto[" \mathrm{~b} " \mapsto " \mathrm{f} ", " \mathrm{a} " \mapsto " \mathrm{i} "], p \mapsto n]\rangle \mid n>0\}
$$


The concretization operation cannot be implemented in a meaningful way, as materializing this infinite set is clearly impossible.

(2) Nondeterminism Not all framework operations will be deterministic, even with a priori knowledge about the program's runtime environment. For example, the exact user input entered is impossible to know at analysis time. Instead, combined interpretation based on $C_{0}$ would have to enumerate over all possible values produced in all environments which is impractical from an implementation perspective.

(3) Exponential Explosion in States Nondeterministic fops whose denotations return multiple possible results will cause exponential explosions in the number of reaching states. For example, if $n$ states reach an fop that produces $m$ unique results, the concrete semantics will generate $n \cdot m$ result states. This problem is similar to the exponential state explosion common in symbolic execution.

Thus, instead of combining abstract and concrete interpretation, CONCERTO combines abstract and mostly-concrete interpretation, which addresses the above limitations. Section 4.2 describes the semantics of mostly-concrete interpretation, Section 4.3 describes how to combine abstract and mostly-concrete interpretation soundly, and finally Section 4.4 gives our precision result for combined interpretation.

\subsection{Mostly-Concrete Interpretation}

Mostly-concrete interpretation introduces the following extensions to concrete interpretation:

Extension 1: The mostly-concrete interpreter supports runtime values that are finite abstractions of (potentially infinite) sets of values. For example, the abstract value $\{+\}$ from Section 2 is a finite abstraction of an infinite set of numbers. Thus, when converting from an abstract to mostly-concrete state, CONCERTO does not need to materialize an infinite (or unmanageably large) set of concrete values; the abstract interpretation need only provide these abstractions.

Extension 2: When an fop would yield an infinite or unmanageably large set of values, the mostly-concrete interpreter may instead use a special "unknown" value that represents "any possible value." The mostly-concrete semantics extend the concrete semantics to soundly handle this value.

Extension 3: The mostly-concrete semantic domain is a map from flow edges to a single mostlyconcrete state, which itself maps program variables to the abstractions of multiple possible values mentioned in Extension 1 above. Thus, our mostly-concrete domain cannot track relationships between program variables, i.e., it is non-relational.

Except for these extensions, the mostly-concrete semantics mirror the concrete semantics (hence the name). Provided all fops are deterministic and environment agnostic, ${ }^{4}$ given a deterministic input state, the results of mostly-concrete and concrete interpretation on framework code will coincide.

To represent multiple possible values for variables of type $\mathcal{F}$, the mostly-concrete domain uses powersets of values in $V_{f}$ extended with a special "unknown" value. For $\mathcal{A}$, the abstract interpretation provides an abstraction domain $\widehat{A}$ that satisfies the properties described in Section 4.3. Informally, the $\widehat{A}$ domain must be non-relational and path-insensitive. However, the abstract value domain used internally by the AI has no such restriction.

Formally, the domain of reaching mostly-concrete states $\widetilde{R}$ is:

$$
\widetilde{R}=\mathcal{L} \rightarrow \widetilde{S} \quad \widetilde{S}=\left(X_{f} \rightarrow \wp\left(V_{f}\right)^{\top}\right) \times\left(X_{a} \rightarrow \widehat{A}\right)
$$

As in Example 3.2, $X_{f}$ and $X_{a}$ are the sets of program variables with types in $\mathcal{F}$ and $\mathcal{A}$ respectively. $\wp\left(V_{f}\right)^{\top}$ is the powerset domain of concrete values in $\mathcal{F}$, extended with $\mathrm{T}_{F}$, which is the "unknown"

\footnotetext{
${ }^{4}$ We say an fop is environment agnostic if it does not depend on the environment model, i.e., $\forall e_{1}, e_{2} \in \mathcal{E}, v_{1}, \ldots, v_{n} \in$ $V_{f} .\left\{v \mid\left\langle v, \_\right\rangle \in \llbracket f o p \rrbracket\left(e_{1}, v_{1}, \ldots, v_{n}\right)\right\}=\left\{v \mid\left\langle v, \_\right\rangle \in \llbracket f o p \rrbracket\left(e_{2}, v_{1}, \ldots, v_{n}\right)\right\}$
} 
value described in Extension 2. The abstractions for variables with types in $\mathcal{A}$ in the mostlyconcrete interpreter are drawn from the domain $\widehat{A}$ provided by the $\mathrm{AI} ; \widehat{A}$ is assumed to form a complete lattice. $\widehat{A}$ may or may not be used internally by the abstract interpreter. The domain of $\widetilde{S}$ and $\widetilde{R}$ form a complete lattice equipped with the standard pointwise join and ordering operators, as well as top and bottom values. We further assume that the analysis defines a monotone complete join-morphism $\alpha_{v}: \wp\left(V_{a}\right) \rightarrow \widehat{A}$ that abstracts a set of concrete values of type $\mathcal{A}$ to a value in $\widehat{A}$.

Example 4.1 (Signedness Analysis). For the signedness analysis, $\widehat{A}$ is the same domain as used in the abstract interpretation, $\wp(\{-, 0,+\}) . \alpha_{v}$ is defined as: $\alpha_{v}(I)=\{\operatorname{sign}(i) \mid i \in I\}$ where sign is defined as in Example 3.2. The join and ordering operators are set union and inclusion respectively. In the example from Section 2, ConCERTo used the abstract value $\{+\}$ for the value of arg in dispatch which abstracted the set $\{n \mid n>0\}$.

Example 4.2 (Pentagons). Suppose instead the abstract interpretation of Section 2 had used a relational domain like Pentagons [Logozzo and Fähndrich 2008], which is the interval domain complemented with symbolic upper bounds. Then, $\widehat{A}=I n t v$, where Int $v$ is the plain interval domain [Cousot and Cousot 1977]. $\alpha_{v}$ is defined as $\alpha_{v}(I)=[l b I, u b I]$ (or $\perp$ if $I=\emptyset$ ), where $u b$ and $l b$ return the upper (resp. lower) bound of a set, or $\infty$ (resp. $-\infty$ ) if no such bound exists in $\mathbb{Z}$. The join and ordering operators are the standard interval union and inclusion operators. Unlike the above example, we cannot reuse the pentagon domain for $\widehat{A}$ for reasons discussed in Section 4.3.

We now define an abstraction function $\alpha_{F}$ as follows:

$$
\alpha_{F}(r)[\ell]=\left\langle\lambda x: X_{f} \cdot \mathcal{V}(r[\ell], x), \lambda x: X_{a} \cdot \alpha_{v}(\mathcal{V}(r[\ell], x))\right\rangle
$$

Where $\mathcal{V}$ is the reaching value set for a set of states and variable, defined as: $\mathcal{V}(S, x)=\{s[x] \mid$ $\left.\left\langle s,{ }_{-}\right\rangle \in S \wedge s[x] \neq \perp_{V}\right\} . \alpha_{F}$ approximates a variable $x$ of type $\mathcal{F}$ with exactly the set of (initialized) values that reach $x$. Variables of type $\mathcal{A}$ are approximated by applying $\alpha_{v}$ to the set of reaching values. The approximation for a variable may not depend on the value of other variables, nor the location at which the variable is being approximated.

As $\alpha_{v}$ is a complete join morphism, $\alpha_{F}$ is also a complete join-morphism, and thus by [Cousot and Cousot 1979b], there exists some $\gamma_{F}$ such that $R \underset{\alpha_{F}}{\stackrel{\gamma_{F}}{\leftrightarrows}} \widetilde{R}$.

In Fig. 7, we define the mostly-concrete semantic function $I_{\top}: \widetilde{R} \rightarrow \widetilde{R}$. The structure of $I_{\top}$ closely mirrors that of the concrete semantic function $F$. Operations and comparisons on values from types in $\mathcal{F}$ use the same operations as in $F$, but lifted to powersets of values. If one of the operands to a comparison is unknown (i.e., $\top_{F}$ ), then both branches are taken. The lifted version of $\llbracket f_{o p} \rrbracket$ in Fig. 7 assumes that if any argument of an fop is unknown (again, $T_{F}$ ) then the result is also unknown. Otherwise, the lifted version computes the result of applying the fop to every possible valuation of arguments in any environment. This can yield large (or even infinite sets) but the semantics of Fig. 7 do not abstract these sets to the $T_{F}$ value, which simplifies our formal presentation. In practice, our implementation falls back on $T_{F}$ for infinite to unmanageably large sets of values (Section 7)

Like concrete interpretation, mostly-concrete interpretation can exploit application-specific knowledge and precisely model fops that would otherwise be treated as nondeterministic. As described in Section 3.1, we model effectively deterministic fops by introducing hypotheses on the domain of program environments $\mathcal{E}$. Thus, despite taking the union over $\mathcal{E}$, the definition of $\widetilde{\llbracket f o p \rrbracket}$ uses only environment models consistent with application-specific information.

Unlike fops, operations on values of types from $\mathcal{A}$ are modeled with imprecise, albeit sound, semantics. Mostly-concrete interpretation can support arbitrary abstractions of $\mathcal{A}$ values precisely because it makes no attempt to interpret aops and therefore does not need to "understand" the 


$$
\begin{aligned}
& I_{\top}(\widetilde{r})\left[\ell^{\circ} \leadsto \ell^{\bullet}\right]=\operatorname{step}^{\top}\left(\bigsqcup_{p \in \operatorname{pred}(\ell)} \widetilde{r}\left[p^{\bullet} \leadsto \ell^{\circ}\right], \ell\right) \sqcup\left\{\begin{array}{ll}
\operatorname{step}^{\top}\left(\perp_{\widetilde{S}}, \ell\right) & \ell=s \\
\perp_{\widetilde{S}} & \text { o.w. }
\end{array} \quad I_{\top}(\widetilde{r})\left[p^{\bullet} \leadsto \ell^{\circ}\right]= \begin{cases}\tilde{r}[p] & \overline{\mathcal{F} \mathcal{T}}\left(\widetilde{r}, p^{\bullet} \sim \ell^{\circ}\right) \\
\perp & \text { o.w. }\end{cases} \right. \\
& \widetilde{\mathcal{F} \mathcal{T}}\left(\widetilde{r}, p^{\bullet} \leadsto \ell^{\circ}\right)= \begin{cases}\widetilde{s}[x] \widetilde{\llbracket<=>\rrbracket} \widetilde{s}[y] & p \in \ell_{f} \wedge \operatorname{prog}[p]=\text { if } x<=>y \text { goto } \ell \\
\widetilde{s}[x] \widetilde{\llbracket<\neq>\rrbracket} \widetilde{s}[y] & p \in \ell_{f} \wedge \operatorname{prog}[p]=\text { if } x<=>\text { goto } \ell^{\prime} \\
\text { true } & \text { o.w. }\end{cases} \\
& \text { Where } \widetilde{s}=\widetilde{r}\left[p^{\circ} \leadsto p^{\bullet}\right] \text { and } \widetilde{\llbracket R \rrbracket} \text { is the lifting of } R \text { defined by: } \\
& \widetilde{v \llbracket R \rrbracket} \top_{F} \quad \top_{F} \widetilde{\llbracket R \rrbracket} \widetilde{v} \quad v \in \widetilde{v} \wedge v^{\prime} \in \widetilde{v^{\prime}} \wedge v \llbracket R \rrbracket v^{\prime} \Rightarrow \widetilde{v} \widetilde{\llbracket R \rrbracket} \widetilde{v}^{\prime}
\end{aligned}
$$

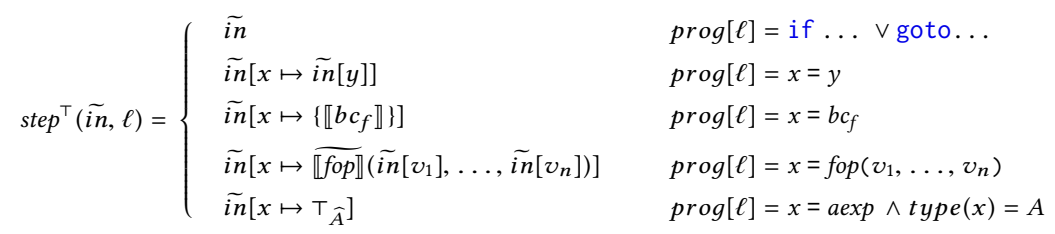

$$
\begin{aligned}
& \widetilde{\llbracket f o p \rrbracket}\left(\widetilde{f}_{1}, \ldots, \tilde{f}_{n}\right)= \begin{cases}T_{F} & \widetilde{f}_{1}=\top_{F} \vee \ldots \vee \widetilde{f_{n}}=\top_{F} \\
\bigcup_{f_{1} \in \tilde{f}_{1}, \ldots, f f_{n} \in \widetilde{f_{n}}}\left\{f^{\prime} \mid\left\langle f^{\prime},{ }_{-}\right\rangle \in \llbracket f o p \rrbracket\left(E, f_{1}, \ldots, f_{n}\right)\right\} & \text { o.w. }\end{cases}
\end{aligned}
$$

Fig. 7. Mostly-concrete semantic function. In $I_{\top}, s$ is again the distinguished program start label.

$$
\begin{aligned}
& \bar{R}=\left(\mathcal{L}_{F} \rightarrow \widetilde{S}\right) \times\left(\mathcal{L}_{A} \rightarrow \widehat{S}\right) \\
& \alpha_{C}(r)=\left\langle\lambda \vec{\ell}: \mathcal{L}_{F} \cdot \alpha_{F}(r)[\vec{\ell}], \lambda \vec{\ell}: \mathcal{L}_{A} \cdot \alpha_{A}(r)[\vec{\ell}]\right\rangle \\
& \widetilde{\tau}: \widehat{S} \rightarrow \widetilde{S} \text { such that: } \forall \vec{\ell}, r: R \cdot \alpha_{F}(r)[\vec{\ell}] \sqsubseteq_{\widetilde{R}} \widetilde{\tau}\left(\alpha_{A}(r)[\vec{\ell}]\right) \\
& \widetilde{i n j}: \bar{R} \rightarrow \widetilde{R} \text { where: } \widetilde{i n j}(\langle\widetilde{m}, \widehat{m}\rangle)[\vec{\ell}]= \begin{cases}\widetilde{m}[\vec{\ell}] & \vec{\ell} \in \mathcal{L}_{F} \\
\widetilde{\tau}(\widehat{m}[\vec{\ell}]) & \vec{\ell} \in \mathcal{L}_{A}\end{cases} \\
& \widehat{\tau}: \widetilde{S} \rightarrow \widehat{S} \text { such that: } \forall \vec{\ell}, r: R \cdot \alpha_{A}(r)[\vec{\ell}] \sqsubseteq_{\widehat{S}} \widehat{\tau}\left(\alpha_{F}(r)[\vec{\ell}]\right) \\
& \widehat{i n j}: \bar{R} \rightarrow \widehat{R} \text { where: } \widehat{i n j}(\langle\bar{m}, \widehat{m}\rangle)[\vec{\ell}]= \begin{cases}\widehat{m}[\vec{\ell}] & \vec{\ell} \in \mathcal{L}_{A} \\
\widehat{\tau}(\widehat{m}[\vec{\ell}]) & \vec{\ell} \in \mathcal{L}_{F}\end{cases}
\end{aligned}
$$

Fig. 8. The combined mostly-concrete and abstract domain definitions, along with the domain transformers and derived injection functions.

abstraction domain $\widehat{A}$. However, CONCERTO does not suffer a precision loss from these coarse semantics; due to the state separation hypothesis, all $a$ ops, $b c_{a}$ and comparisons over $\mathcal{A}$ occur in statements labeled $\ell_{a}$, which are modeled in CONCERTO with abstract interpretation. In other words, the mostly-concrete semantics for operations over $\mathcal{A}$ values are imprecise, but never actually executed in the mostly-concrete interpreter.

Theorem 2. $\alpha_{F} \circ F \sqsubseteq_{R \rightarrow \widetilde{R}} I_{\top} \circ \alpha_{F}$, i.e., $I_{\top}$ is a sound over-approximation of $F$.

Proof Sкетсн. By case analysis on the definitions of step $^{F}$ and step $p^{\top}$. The full proofs are available in Appendix A.1.

\subsection{Combined Abstract and Mostly-Concrete Interpretation}

We now show how to combine the mostly-concrete and abstract interpreters. The approach broadly mirrors the strawman approach from Section 4.1. Specifically, combined interpretation operates over a combined domain $\bar{R}$. Like its strawman counterpart $\bar{R}_{0}, \bar{R}$ represents reaching states in the application with abstract state $\widehat{S}$, but uses mostly-concrete states $\widetilde{S}$ for the framework instead of $\wp(S)$. CONCERTO's combined interpretation also injects the combined state representation into the "native" formats expected by the abstract and mostly-concrete interpreters. However, instead of using abstraction and concretization functions as in Section 4.1, we use domain transformers to 
soundly translate between state representations without requiring one of the abstract or mostlyconcrete state representation to be more precise than the other.

The combined analysis domain $\bar{R}$ is defined in Fig. 8, along with an abstraction function $\alpha_{C}$. As $\alpha_{A}$ and $\alpha_{F}$ are complete join morphisms, $\alpha_{C}$ is itself a complete join morphism, and thus there exists some $\gamma_{C}$ such that $R$ and $\bar{R}$ form a Galois connection. The monotone functions $\widetilde{\tau}$ and $\widehat{\tau}$ in Fig. 8 are the domain transformers described above: $\widetilde{\tau}$ transforms a state from the abstract interpreter into a mostly-concrete state, and $\widehat{\tau}$ performs a transformation in the opposite direction. They are both functions provided by the analysis that must fulfill the following conditions:

$$
\forall \vec{\ell}, r: R \cdot \alpha_{A}(r)[\vec{\ell}] \sqsubseteq \widehat{s} \widehat{\tau}\left(\alpha_{F}(r)[\vec{\ell}]\right)
$$

$$
\forall \vec{\ell}, r: R \cdot \alpha_{F}(r)[\vec{\ell}] \sqsubseteq_{\widetilde{S}} \widetilde{\tau}\left(\alpha_{A}(r)[\vec{\ell}]\right)
$$

Intuitively, conditions (12) and (13) state that the transformers must be consistent with the target domain's abstraction function. As a consequence, (13) implies that any relational information present in $\widehat{R}$ must be discarded when moving to the non-relational domain $\widetilde{R}$. The $\widehat{A}$ values produced by $\alpha_{F}$ are the result of a non-relational abstraction function $\alpha_{v}$, and by the inequality of Eq. (13), the result of $\widetilde{\tau}$ can do no better. Despite this restriction on relational abstractions in the mostly-concrete domain, the above requirements on the the domain transformers do not provide information about the relative precision of the two domains. In fact, as mentioned above and illustrated below, it may not necessarily be the case that one of the domains is more precise than the other.

Example 4.3 (Trivial Transformers). Consider the domain of Example 3.2 and the definition of $\alpha_{v}$ from Example 4.1. The two state representations are equal $(\widehat{S}=\widetilde{S})$, and $\widetilde{\tau}=\widehat{\tau}=i d$. In other words, the two domains have the same expressive power.

Example 4.4 (Relational Domain). Suppose instead of representing integers with of the signedness domain used in Example 3.2, we used the Pentagon domain of Example 4.2 with the corresponding $\alpha_{v}$ and $\widehat{A}=I n t v$. Then the abstract domain is $X_{a} \rightarrow \operatorname{Int} v \times X_{a} \rightarrow \wp\left(X_{a}\right) \times X_{f} \rightarrow \wp\left(V_{f}\right)^{\top}$, where the first two components are respectively the interval environment and strict upper bounds of integers described in [Logozzo and Fähndrich 2008]. This abstract domain has used the $\wp\left(V_{f}\right)^{\top}$ representation for framework variables. As with the embedding of $\widehat{A}$ in the mostly-concrete interpreter, this embedding of mostly-concrete values into the abstract domain is feasible due to the state separation hypothesis: i.e., fops will not appear in code analyzed by the abstract interpreter.

The domain transformers may be defined as:

$$
\tilde{\tau}(\langle b, s, m\rangle)=\langle b, m\rangle \quad \widehat{\tau}(\langle b, m\rangle)=\left\langle b, \lambda x: X_{a} \cdot \emptyset, m\right\rangle
$$

That is, $\widetilde{\tau}$ discards the relational information from $\widehat{S}$ when moving to $\widetilde{S}$, and $\widehat{\tau}$ uses the top element of the strict upper bound domain in the output (as the input mostly-concrete state does not have any relational information). In this example, the abstract domain is more precise, i.e. $\widehat{S} \underset{\tau}{\stackrel{\tau}{\tau}} \widetilde{S}$.

Example 4.5 (Trivial Abstract Domain). Consider a domain $X_{a} \rightarrow \wp(\{-, 0,+\}) \times X_{f} \rightarrow \mathbf{1}$, where $\mathbf{1}$ is unary domain whose single element $t t$ represents "any possible value", i.e., the analysis does not try to reason about maps, strings, or I/O. Then $\widetilde{\tau}(\langle m, z\rangle)=\left\langle m, \lambda x: X_{f} \cdot \top_{F}\right\rangle$ and $\widehat{\tau}(\langle m, t\rangle)=\langle m, \lambda x$ : $\left.X_{f} . t \mathrm{t}\right\rangle$, and $\widetilde{S} \underset{\widehat{\tau}}{\stackrel{\tau}{\tau}} \widehat{S}$, i.e., the mostly-concrete domain is more precise than the abstract domain.

Example 4.6 (Mixed Expressiveness). Finally, consider a combination of Examples 4.4 and 4.5 where integers (i.e., variables in $X_{a}$ ) are modeled in the abstract domain with pentagons, $\widehat{A}=\operatorname{Int} v$, but framework types (maps and strings) are modeled with the highly imprecise domain $\mathbf{1}$. Then $\widetilde{\tau}(\langle b, s, z\rangle)=\left\langle b, \lambda x: X_{f} \cdot \top_{F}\right\rangle$ and $\widehat{\tau}(\langle b, m\rangle)=\left\langle b, \lambda x: X_{a} \cdot \emptyset, \lambda x: X_{f} . t \mathrm{t}\right\rangle \cdot \widetilde{\tau}$ and $\widehat{\tau}$ do not form a 


$$
\begin{aligned}
& L B(r)\left[\ell^{\circ} \leadsto \ell^{\bullet}\right]=\operatorname{step}^{L B}\left(\sqcup_{p \in \operatorname{pred}(\ell)} r\left[p^{\bullet} \leadsto \ell^{\circ}\right], \ell\right) \sqcup \begin{cases}\operatorname{step}^{L B}\left(\perp_{\tilde{S}}, s\right) & \ell=s \\
\perp_{\widetilde{S}} & \text { o.w. }\end{cases} \\
& L B(r)\left[\ell_{f}^{\bullet} \leadsto \ell^{\circ}\right]=r\left[\ell_{f}^{\circ} \leadsto \ell_{f}^{\bullet}\right] \quad L B(r)\left[\ell_{a}^{\bullet} \leadsto \ell^{\circ}\right]=\perp
\end{aligned}
$$

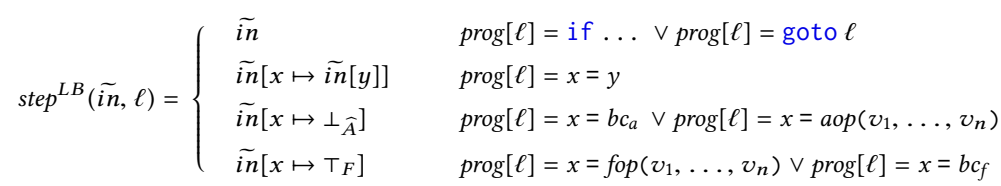

Fig. 9. Semantic function defining a lower-bound on the precision of the abstract interpretation.

Galois connection: the abstract domain is more precise for integers, but the mostly-concrete domain more precisely represents maps and strings.

We are ready to define the combined interpretation function $C: \bar{R} \rightarrow \bar{R}$ as follows:

$$
C(X)=\left\langle\left. I_{\top} \circ \widetilde{\operatorname{inj}}(X)\right|_{\mathcal{L}_{F}},\left.\widehat{F} \circ \widehat{\operatorname{inj}}(X)\right|_{\mathcal{L}_{A}}\right\rangle
$$

This definition closely mirrors Eq. (1). The injection functions $\widetilde{i n j}$ and $\widehat{i n j}$ (defined in Fig. 8) take the place of $i n j_{R}$ and $i n j_{\widehat{R}}$ and translate the combined domain into $\widetilde{R}$ and $\widehat{R}$ by using the domain transformers $\widetilde{\tau}$ and $\widehat{\tau}$ respectively. The fully-concrete semantic function $F$ has also been replaced with the mostly-concrete semantic function $I_{\mathrm{T}}$.

We can state the soundness of $C$ according to $\alpha_{C}$ :

Theorem 3. $\alpha_{C} \circ F \sqsubseteq_{R \rightarrow \bar{R}} C \circ \alpha_{C}$

Proof Sketch. By Theorem 2, the assumed soundness of $\widehat{F}$, and from the fact that $\alpha_{F} \sqsubseteq \widetilde{i n j} \circ \alpha_{C}$ and that $\alpha_{A} \sqsubseteq \widehat{i n j} \circ \alpha_{C}$.

As $C$ is a monotone function on a complete lattice it has a least fixed point [Tarski 1955]. From Theorem 3 and [Cousot and Cousot 1979b] we then have that: $\alpha_{C}(l f p F) \sqsubseteq l f p C$.

\subsection{Conditions for Increased Precision}

$C$ is sound, but may not necessarily be more precise than the original function $\widehat{F}$. We now discuss a set of sufficient conditions for when $C$ is at least as (if not more) precise as $\widehat{F}$.

First, we define a function $L B: \widetilde{R} \rightarrow \widetilde{R}$ as shown in Fig. 9. Intuitively, $L B$ provides a lowerbound on the precision of the abstract semantic function; showing that $I_{\top}$ can "do better" than this lower bound will imply that $I_{\top}$ provides improved precision compared to $\widehat{F}$ on framework code. $L B$ specifies an imprecise lower bound for modeling framework operations and comparisons, but provides no lower bound on the precision for application operations or comparisons. However, as $L B$ is non-relational, the definition implies that $\widehat{F}$ must also be non-relational.

$C$ is more precise (as defined below) if the following conditions hold:

$$
\widehat{\tau} \circ \tilde{\tau}=i d \quad L B \circ \dot{\tilde{\tau}} \sqsubseteq_{\widehat{R} \rightarrow \widetilde{R}} \dot{\tilde{\tau}} \circ \widehat{F} \quad \forall U \subseteq \widehat{S} . \widetilde{\tau}(\sqcup U)=\sqcup_{\widehat{s} \in U} \widetilde{\tau}(\widehat{s})
$$

That is, if no precision is lost by moving to the domain $\widetilde{S}$ and then back to $\widehat{S}$, if $L B$ is a lower bound on the precision of $\widehat{F}$, and if $\widetilde{\tau}$ is a complete join morphism. In the above, $\dot{\tilde{\tau}}$ denotes the pointwise extension of $\widetilde{\tau}$.

As $C$ and $\widehat{F}$ operate over different domains, we first introduce a function $\widehat{p r o j}: \widehat{R} \rightarrow \bar{R}$ to project the $\widehat{R}$ domain into the combined domain $\bar{R}$ :

$$
\widehat{\operatorname{proj}}(\widehat{s})=\left\langle\lambda \vec{\ell}: \mathcal{L}_{F} \cdot \widetilde{\tau}(\widehat{s}[\vec{\ell}]), \lambda \vec{\ell}: \mathcal{L}_{A} \cdot \vec{s}[\vec{\ell}]\right\rangle
$$


$\widehat{\text { proj }}$ uses $\widetilde{\tau}$ to translate abstract to mostly-concrete states, reversing the $\widehat{i n j}$ operation.

If the above conditions hold, we can prove:

Theorem 4. $\widehat{\operatorname{inj}}(l f p C) \sqsubseteq_{\widehat{R}} l f p \widehat{F}$

Proof Sketch. From the assumptions in Eq. (19) and by case analysis on $s t e p^{\top}$ and $s t e p^{L B}$, it can be shown that $C \circ \widehat{\operatorname{proj}} \sqsubseteq_{\widehat{R} \rightarrow \bar{R}} \widehat{\operatorname{proj}} \circ \widehat{F}$ (Lemma 6 in the appendix), whence it follows by straightforward transfinite induction (Lemma 7 in the appendix) that $l f p C \sqsubseteq_{\bar{R}} \widehat{\operatorname{proj}}(l f p \widehat{F})$. As $\widehat{i n j}$ is monotone, we have: $\widehat{i n j}(l f p C) \sqsubseteq_{\widehat{R}} \widehat{i n j} \circ \widehat{\operatorname{proj}}(l f p \widehat{F})$, whence we have $\widehat{i n j}(l f p C) \sqsubseteq_{\widehat{R}} l f p \widehat{F}$, as $\widehat{i n j} \circ \widehat{p r o j}=i d$ by Eq. (19).

Theorem 4 states that the approximation of reaching states computed by the combined interpretation function $C$ is at least as precise as that computed by the abstract interpreter. The inequality is not strict: whether CONCERTO matches or exceeds the precision of plain abstract interpretation depends on the program and the abstract semantics and domain. As a trivial example, on a program with only application statements (i.e., with labels drawn from $\mathcal{L}_{A}$ ) CONCERTO will necessarily do no better than plain abstract interpretation.

Example 4.7 (Signedness Analysis). The signedness example of Section 2 satisfies the above conditions, and thus combined interpretation with CONCERTO is at least as precise as plain abstract interpretation. As both $\widetilde{\tau}$ and $\widehat{\tau}$ are the identity function, parts 1 and 3 of Eq. (19) are trivially satisfied. The abstract semantic function in Fig. 6 uses very coarse approximations of fop operations, and therefore part 2 of Eq. (19) is also satisfied.

\section{PROCEDURES}

Our formalism so far does not support procedures, but they are essential. Section 5.1 sketches the addition of procedures to our formal language and extensions to the concrete and mostlyconcrete semantics. Section 5.2 then demonstrates that by restricting control transfers between the framework and application to procedures calls and returns, CONCERTO does not need AI developers to provide the full domain transformers $(\widetilde{\tau}$ and $\widehat{\tau})$ introduced in Section 4.3 since it suffices to transform only parameter and return values across procedure boundaries. We delay until Section 8 how to extend our support for procedures to objects and methods in the style of Java.

\subsection{Interprocedural Semantics}

We assume a program now has the following form, where $f$ ranges over procedure names:

$$
\begin{aligned}
& \operatorname{prog}::=D * \quad \text { astmt }::=\ldots \mid \text { return } x \mid x=f(y) \\
& D::=\operatorname{proc} f(p)\left\{\left(f s t m t^{\ell} \mid \text { astmt }^{\ell}\right)^{*}\right\} \quad f \text { stmt }::=\ldots \mid \text { return } x \mid x=f(y)
\end{aligned}
$$

We assume that procedure names are distinct, that there is a distinguished main procedure, and that the distinguished program start label $s$ is the first statement of this procedure.

For a procedure call statement labeled with $\ell$, we assume there are two pseudo-labels: $\ell_{c}$ and $\ell_{r}$, corresponding to the point in program execution immediately before invoking the function and immediately after the called function returns. We extend the succ and pred relations to include these pseudo-labels. For a call-site labeled $\ell$, if control may flow from label $\ell^{\prime}$ to $\ell,\left(\ell^{\prime}, \ell_{c}\right) \in$ succ, and similarly for $\left(\ell_{r}, \ell^{\prime}\right)$ and control-flow from $\ell$ to $\ell^{\prime}$. In addition, the entry point label of the callee at $\ell$ is a successor of $\ell_{c}$ and the predecessor of $\ell_{r}$ is the return site label in the callee.

We extend the state definition to track the runtime stack: $R=\mathcal{L} \rightarrow \wp\left(S^{*} \times \ell^{*} \times \mathcal{E}\right)$, where $S^{*}$ is a sequence of states $S$ as defined in Section 3.2, and $\ell^{*}$ is a sequence of statement labels. Intuitively, these two components represent the runtime stack and the return locations for active procedure invocations respectively. We also assume that the domain of variables $X$ includes a special return slot $\rho$. 


$$
\begin{aligned}
& F(r)\left[\ell_{r}^{\circ} \leadsto \ell_{r}^{\bullet}\right]=\bigsqcup_{p \in \operatorname{pred}\left(\ell_{r}\right)}\left\{\left\langle s \circ s_{r}\left[x \mapsto s_{c}[\rho]\right], \mathcal{R}, E\right\rangle \mid\right. \\
& \left\langle s \circ s_{r} \circ s_{c}, \mathcal{R}, E\right\rangle \in r\left[p^{\bullet} \sim \ell_{r}^{\circ}\right] \wedge \\
& \left.\left\langle s \circ s_{r}, \mathcal{R},{ }_{-}\right\rangle \in r\left[\ell_{c}^{\circ} \sim \ell_{c}^{\bullet}\right]\right\} \\
& F(r)\left[p^{\bullet} \leadsto \ell_{r}^{\circ}\right]=\{\langle s, \mathcal{R}, E\rangle \mid\langle s, \mathcal{R} \circ \ell, E\rangle \in r[p]\} \\
& F(r)\left[\ell_{c}^{\bullet} \leadsto \ell^{\prime \circ}\right]=\left\{\left\langle s \circ s_{r} \circ \iota_{S}\left[p \mapsto s_{r}[y]\right], \mathcal{R} \circ \ell, E\right\rangle \mid\right. \\
& \left.\left\langle s \circ s_{r}, \mathcal{R}, E\right\rangle \in r\left[\ell_{c}\right]\right\} \\
& F(r)\left[\ell_{c}^{\circ} \leadsto \ell_{c}^{\bullet}\right]=\bigsqcup_{p \in \operatorname{pred}\left(\ell_{c}\right)} r\left[p^{\bullet} \leadsto \ell_{c}^{\circ}\right]
\end{aligned}
$$

$$
\begin{aligned}
& I_{\top}(\widetilde{r})\left[\ell_{r}^{\circ} \leadsto \ell_{r}^{\bullet}\right]=\bigsqcup_{p \in \operatorname{pred}\left(\ell_{r}\right)} \tilde{r}\left[\ell_{c}\right]\left[x \mapsto \widetilde{r}\left[p^{\bullet} \leadsto \ell_{r}^{\circ}\right][\rho]\right] \\
& I_{\top}(\widetilde{r})\left[p^{\bullet} \leadsto \ell_{r}^{\circ}\right]=\widetilde{r}[p] \\
& I_{\top}(\widetilde{r})\left[\ell_{c}^{\bullet} \leadsto \ell^{\prime \circ}\right]=\left[p \mapsto \widetilde{r}\left[\ell_{c}\right][y]\right] \\
& I_{\top}(\widetilde{r})\left[\ell_{c}^{\circ} \leadsto \ell_{c}^{\bullet}\right]=\bigsqcup_{p \in \operatorname{pred}\left(\ell_{c}\right)} \widetilde{r}\left[p^{\bullet} \leadsto \ell_{c}^{\circ}\right] \\
& \operatorname{step}^{\top}(\tilde{\operatorname{in}}, \ell)= \begin{cases}\tilde{\operatorname{in}}[\rho \mapsto \tilde{\operatorname{in}}[x]] & \operatorname{prog}[\ell]=\operatorname{return} x \\
\text { as before } & \text { o.w. }\end{cases} \\
& \operatorname{step}^{F}(\text { in }, \ell)= \begin{cases}\text { in }[\rho \mapsto \operatorname{in}[x]] & \text { prog }[\ell]=\operatorname{return} x \\
\text { as before } & \text { o.w. }\end{cases}
\end{aligned}
$$

Fig. 10. Extensions to the semantic functions $F$ and $I_{\top}$ to support procedures. In the above definitions, $p$ is the name of the parameter of the called procedures, $y$ is the variable passed as the argument, and $x$ is the variable in the caller to which the result of the function is defined. $\iota_{S}$ is a state where all variables are bound to $\perp_{V}$.

We extend the definitions of $F$ and $I_{\top}$ given in Sections 3.2 and 4.2 respectively as shown in Fig. 10 to handle these new statement forms and flow edges. We omit the updated definitions of $F$ and $I_{\mathrm{T}}$ at the distinguished start label, although they are the obvious extensions to the terms in Figs. 5 and 7. We use $\rho$ to store the return value of functions with return type $\mathcal{F}$ and $\mathcal{A}$ : we shall assume that there are two different versions of $\rho$ of the appropriate type. Finally, $\mathcal{V}$ is now defined as: $\mathcal{V}(\mathcal{S}, x)=\left\{s[x] \mid\left\langle s_{0} \circ s,{ }_{-}\right\rangle \in \mathcal{S} \wedge s[x] \neq \perp_{V}\right\}$.

We have proved (see Appendix B) that this updated definition of $I_{\top}$ is sound with respect to the updated definition of $F$. If the abstract interpretation is also sound with respect to this updated definition, and the $\widetilde{\tau}$ and $\widehat{\tau}$ functions still fulfill the conditions in Section 4 then all the results of the previous section still hold with no modifications to our formalisms or proofs.

\subsection{Interprocedural Domain Transformers}

We have so far treated procedure call and return as orthogonal to control-flow transfer between the application and framework, i.e., procedure bodies could be a $\operatorname{mix} \ell_{f}$ and $\ell_{a}$ statements. In practice, we require all transfers between the application and framework occur at procedure boundaries. In other words, each procedure may only contain $\ell_{a}$ statements or $\ell_{f}$ statements. This restriction is entirely reasonable: real frameworks encapsulate their functionality in methods/classes/modules/etc.: source programs would not mix framework and application code in the same procedure.

This (non-)restriction also significantly simplifies how CONCERTO transfers values between abstract and mostly-concrete interpretation. Recall that CONCERTO applies the domain transformers at transitions between the application and framework. However, the above syntactic restriction implies that CONCERTO needs the domain transformers only at procedure entry and exit. Further, at procedure entry, the mostly-concrete interpreter can access only the program state reachable via the parameters. Thus, instead of using $\widetilde{\tau}$ at application-to-framework calls, the AI may directly provide mostly-concrete arguments to the mostly-concrete component, which then binds the arguments in a empty local state. Similarly, we assume that when given mostly-concrete arguments the abstract interpreter can construct a sound abstract procedure entry state. (This assumption is possible because our language is statically scoped and has no global variables, heaps are discussed in Section 8.) As a consequence, instead of using $\widehat{\tau}$ at framework-to-application calls, the mostly-concrete interpreter may provide mostly-concrete arguments to the AI, which binds them in an abstract empty state, transforming the mostly-concrete values into a "native" abstract representation as necessary.

CONCERTO uses a similar process for return flows. The mostly-concrete semantics use the exit state of a called procedure only to extract the procedure's return value. Instead of using $\widetilde{\tau}$ at return flows from the application to framework, the AI simply provides a mostly-concrete representation of the 
return slot $\rho$. We likewise assume that the AI is interested only in the value of $\rho$ in callee exit states. Thus, at framework-to-application return flows, the mostly-concrete interpreter may provide only the mostly-concrete return value, which the AI transforms into a native representation as necessary.

The direct exchange of values sketched above obviates the need for implementations of $\widetilde{\tau}$ and $\widehat{\tau}$, but to retain soundness, the values exchanged must be consistent with those produced by some $\widetilde{\tau}$ and $\widehat{\tau}$ that satisfy the definitions given in Section 4.3. For example, consider an application-toframework call where the abstract entry state is $\widehat{s}$. The mostly-concrete argument provided to the mostly-concrete interpreter must therefore be $\widetilde{\tau}(\widehat{s})[p]$ for some $\widetilde{\tau}$, and where $p$ is the parameter of the called procedure. Thus, any valid domain transformers $\widetilde{\tau}$ and $\widehat{\tau}$ provide correctness specifications for the exchange of values. However, a trivial way to ensure soundness is to generate complete definitions for some $\widetilde{\tau}$ and $\widehat{\tau}$, and hand-simplify the results of applying the transformers at procedure call and return. For example, to correctly generate mostly-concrete return values at application-toframework return flows, it is sufficient to use a simplification of $\lambda \widehat{s} . \widetilde{\tau}(\widehat{s})[\rho]$.

Context Sensitivity. The above discussion does not treat context-sensitivity in the abstract interpretation. In our implementation we have the AI provide functions to compute the callee context at framework-to-application calls. Further the mostly-concrete semantics in Fig. 10 are contextinsensitive, which is not very precise in practice. In practice, we unroll the call-graph up to recursive cycles in the mostly-concrete interpreter, effectively giving unlimited context-sensitivity. We discuss these two techniques in Section 8.3. We have not formalized our approach to context-sensitivity, although adapting our proofs and formalism is a straightforward, albeit tedious, extension.

\section{ITERATION STRATEGY}

We now briefly describe the iteration strategy used by CONCERTO. Our implementation runs mostlyconcrete and abstract interpreters in parallel until they converge to a local fixed-point on their respective partitions of the program (framework and application code respectively). The results are then exchanged between the two interpreters (under the syntactic restriction of Section 5.2, this exchange is performed at procedure boundaries as described above) and the process repeats until the overall process converges to a fixed-point. We refer to this process as subfixpoint iteration.

The subfixpoint iteration scheme sketched above is very similar to chaotic asynchronous iteration with memory [Cousot 1977], with one key difference. Before starting iteration in the mostlyconcrete component, subfixpoint iteration discards results at framework statements computed in previous rounds of subfixpoint iteration. In other words, after exchanging information with the abstract interpretation component, the mostly-concrete component iterates a "fresh" mostlyconcrete interpreter, beginning with only information received from the abstract interpreter. We have shown in Appendix $C$ that this process converges to the least fixed point of $C$. Our proof depends on the following property of subfixpoint iteration (Lemma 21 in the appendix): any information discarded between runs of the mostly-concrete interpreter can be soundly recovered with enough iterations in the next run of the mostly-concrete interpreter.

This iteration strategy justifies analyzing application-to-framework calls by spawning a fresh mostly-concrete interpreter seeded with mostly-concrete arguments provided by the AI that flow into the framework from the application. We describe this process in more detail in Section 8.

\section{WIDENING AND FINITIZATION}

Two significant challenges remain to a realizable implementation. First, although we have proved that subfixpoint iteration converges to the least fixed point of $C$, it may not do so in finite time; the domain $\bar{R}$ does not possess an ascending chain condition that will ensure convergence in finite steps. Second, we have not yet guaranteed that mostly-concrete interpretation manipulates only 
finite sets of values. To address the first issue, we apply widening [Cousot and Cousot 1977] during iteration. We address the second issue by forbidding infinite sets of values, and describe how the mostly-concrete interpreter uses $T_{F}$ in practice to avoid materializing infinite sets.

Widening. Following the vocabulary of [Bourdoncle 1993], we require two widening point sets $\mathcal{W}_{A}$ and $\mathcal{W}_{F}$ for application and framework statements, respectively. A widening point set is a set of statement labels such that, if during iteration the states at all widening points stabilize, then the overall iteration stabilizes in a finite number of steps. We further require that if the states at all widening points in two iteration sequences stabilize to the same set of values, then the two sequences stabilize to the same result. We leave the choice of $\mathcal{W}_{A}$ up to the abstract interpretation, although we expect most interpreters will use a variation on the strategy described by [Bourdoncle 1993]. In our mostly-concrete interpreter, we use the headers of unbounded loops and the entry point of a representative method selected from recursive cycles (including sub-cycles).

We assume that the analysis provides widening operators $\nabla_{\widehat{A}}$ for values of type $\widehat{A}$ and $\widehat{\nabla}$ for abstract states $\widehat{S}$. From $\nabla_{\widehat{A}}$, we derive a widening operator for mostly-concrete states:

$$
\left\langle m_{f}, m_{a}\right\rangle \widetilde{\nabla}\left\langle m_{f}^{\prime}, m_{a}^{\prime}\right\rangle=\left\langle\left(\lambda x: X_{f} \cdot\left\{\begin{array}{ll}
m_{f} & m_{f}^{\prime}[x] \sqsubseteq m_{f}[x] \\
\top_{F} & \text { o.w. }
\end{array}\right),\left(\lambda x: X_{a} \cdot m_{a}[x] \nabla_{\widehat{\mathrm{A}}} m_{a}^{\prime}[x]\right)\right\rangle\right.
$$

Given the above assumptions and definitions, we ensure termination as follows. We again iterate the abstract and mostly-concrete interpreters in parallel, except we instrument the abstract semantic function $\widehat{F}$ and $I_{\mathrm{T}}$ to apply widening operations at the locations in the widening point sets $\mathcal{W}_{A}$ and $\mathcal{W}_{F}$ respectively. We have proved (Appendix D) that these individual iterations terminate in a finite number of steps. After the two interpreters stabilize, they exchange results and the process repeats; with the mostly-concrete interpreter again discarding previously computed information as described above. This process stabilizes in a finite number of steps to an over-approximation of $l f p C$ (see Appendix D.1).

Precision. Section 4.4 gave conditions for when $l f p C$ will be at least as precise as $l f p \widehat{F}$. Whether this precision result also translates to the widened subfixpoint iteration presented above will depend on the choice of widening operators. As widening operators are not necessarily monotone, the instrumented $\widehat{F}$ and $I_{\top}$ functions are not necessarily monotone either. Without monotonicity, reasoning about the relative precision of subfixpoint iteration with widening is difficult. This result is not surprising; as noted in [Cousot and Cousot 1992a], when using widening the order of iteration can have a significant impact on the precision of the final result.

Finitization. Finally, to ensure CONCERTO manipulates only finite sets, we require that the AI does not provide infinite arguments or return value representations to the mostly-concrete interpreter. We also extend the definition of $I_{\top}$ to finitize the result of all fop operations. Whenever applying fop to two arguments would produce an infinite set of values (or an otherwise impractically large set, e.g., all 32-bit machine integers) the modified semantic function abstracts this set with $\mathrm{T}_{F}$. This finitization is sound, while avoiding materializing infinite sets in our implementation.

\section{EXTENSIONS FOR A REALISTIC PROTOTYPE}

We have implemented a prototype combined interpreter for a subset of Java. ${ }^{5}$ Our subset includes 1) interfaces and dynamic dispatch, 2) reflection, 3) dynamically sized arrays, and 4) (potentially) unbounded loops. We include two primitives to simulate I/O. The first, read(), reads an integer from a deterministic stream. This primitive models reading from a deterministic configuration file. The second primitive, nondet() reads from a nondeterministic stream, which simulates, e.g., packets received from the network or user input. While falling considerably short of the full complexity of

${ }^{5}$ Our implementation prototype is open-source and is available at https://github.com/uwplse/concerto. 
Java, we can use these language features to effectively simulate some of the most difficult to analyze code idioms we have encountered in real-world framework implementations (see Section 9).

Our prototype takes as input an abstract interpretation implementation which exposes basic operations required by CONCERTO. We introduce these operations incrementally as we extend our basic procedural language to support objects, methods, etc.

\subsection{Objects and the Heap}

We first consider only class-based objects with fields, deferring methods to Section 8.2 and primitives, interfaces, and libraries to Section 8.4. We assume each concrete class belongs either to the framework or the application, taking $\mathcal{F}$ to be the framework classes and $\mathcal{A}$ the application classes We also extend our concrete state to include a concrete heap: $S=\mathcal{E} \times(X \rightarrow V) \times H$. Object allocation and field manipulation are defined via aops and fops that additionally side-effect the heap (we omit a full formalization for space reasons). This formulation implies that framework code may not directly manipulate the object fields of application classes and vice versa. However, as argued in Section 2, real-world applications and frameworks almost exclusively communicate via functional interfaces.

By classifying each class as either framework or application, we can effectively partition the program's runtime heap $H$ into $H_{a}$ containing application objects and $H_{f}$ containing framework objects, i.e., $S=\mathcal{E} \times(X \rightarrow V) \times H_{a} \times H_{f}$. We can then use different abstractions for $H_{a}$ and $H_{f}$.

In the mostly-concrete interpreter, object operations on framework classes manipulate mostlyconcrete heaps of type $\widetilde{H_{f}} \cdot{ }^{6} \widetilde{H_{f}}$ is equipped with join, ordering, and widening operators. The mostlyconcrete interpreter does not use its own abstraction for $H_{a}$, using instead the one provided by the abstract interpreter (see below). Similarly, in the abstract interpreter, object operations on application classes operate on an abstract representation of $H_{a}$, i.e., the application heap component. ${ }^{7}$ CoNCERTO does not make any assumptions on the internal heap representation used by the abstract interpretation. However, the abstract interpretation must provide two functions: projectH : $\widehat{S} \rightarrow \widehat{H_{a}}$ and injectH : $\widehat{H_{a}} \rightarrow \widehat{S} \rightarrow \widehat{S}$ such that $\forall \widehat{s} . \widehat{s} \sqsubseteq_{\widehat{S}}$ injectH (projectH $\widehat{s}$ ) $\widehat{s}$ for some type $\widehat{H_{a}}$ defined by the analysis. The AI must provide widening, join, and ordering operations for $\widehat{H_{a}}$.

To tie these two heap representations together, we extend the mostly-concrete state representation to include mostly-concrete and abstract heaps: $\widetilde{S}=\left(X_{f} \rightarrow \wp\left(V_{f}\right)^{\top}\right) \times\left(X_{a} \rightarrow \widehat{A}\right) \times \widetilde{H_{f}} \times \widehat{H_{a}} \cdot V_{f}$ is the domain of mostly-concrete heap locations, and $\widehat{A}$ is an abstract representation of objects of type $\mathcal{A}$. During execution, the mostly-concrete interpreter updates the mostly-concrete heap and threads the abstract heap representation through unchanged. CONCERTO requires that the abstract interpreter also threads the mostly-concrete heap through its interpretation. To ensure the concrete heap is correctly handled, the abstract interpretation must operate over an instrumented state representation, $\widehat{S} \times H_{f}$. We provide APIs for the AI to manipulate this instrumented representation.

The approach described so far does not allow for framework objects to store references to application objects and vice versa. To relax this restriction, we require the abstract interpretation meets some additional conditions. First, the abstract heap must represent fields and variables with type $\mathcal{F}$ with the domain $\wp\left(V_{f}\right)^{\top}$. Next, when performing a write of $v^{\prime}: \wp\left(V_{f}\right)^{\top}$ to a field/variable of type $\mathcal{F}$ with the existing value $v: \wp\left(V_{f}\right)^{\top}$, the value $v^{\prime \prime}$ of the field/variable after the write must satisfy the constraint $v^{\prime \prime} \sqsubseteq v^{\prime} \sqcup v$, i.e., the new value is bounded above by the result from a weak update. This requirement ensures that the abstract interpretation never produces mostly-concrete

\footnotetext{
${ }^{6} \mathrm{We}$ do not use a fully concrete heap to handle object allocations in unbounded loops. Our widening operator detects such cases and introduces mostly-concrete summary objects where appropriate.

${ }^{7}$ Technically, the abstract interpretation may also operate on an abstraction of $H_{f}$. However, all object operations that mutate $H_{f}$ are modeled by the mostly-concrete interpreter, so in practice the abstract interpreter only uses an abstraction of $H_{a}$.
} 
object locations "out of thin air" that may not have yet been allocated in the mostly-concrete heap component. A similar concern exists when storing values of type $\mathcal{A}$ into the concrete heap: any abstractions stored into the concrete heap must remain valid, and updates via aliasing should be propagated to these values. A sufficient condition is for the abstract interpreter to internally use an abstract heap $\widehat{L O C} \rightarrow \widehat{O}$ where $\widehat{L O c}$ is a finite domain of abstract locations and $\widehat{O}$ are abstract objects, and to take $\widehat{A}=\wp(\widehat{L o c})$ and $\widehat{H_{a}}=\widehat{L o c} \rightarrow \widehat{O}$.

\subsection{Methods and Domain Transformers}

We require that methods in application classes contain only application code, and similarly for framework classes. As described in Section 5.2, this (non-)restriction ensures that values change representation only at method boundaries.

For framework-to-application calls, the abstract interpreter must expose a method interpret that analyzes a method $m$ in context $C$ (see below), with mostly-concrete arguments $a_{1}, a_{2}, \ldots, a_{n}$, and abstract receiver $\widehat{r}$. When CONCERTO encounters a method call in the mostly-concrete interpreter with a base pointer $\widehat{r}$ of type $\widehat{A}$ (i.e., static type $\mathcal{A}$ ), it yields into the abstract interpreter by passing the mostly-concrete arguments, abstract receiver, and a computed context $C$ to interpret. interpret is responsible for constructing an initial abstract state for $m$ and then performing abstract interpretation over the method body. When analysis of $m$ is complete, interpret returns a mostly-concrete representation of the return value. This process mirrors the one described in Section 5.2. However, CONCERTO additionally instruments the above process to inject the caller's mostly-concrete and abstract heaps into the callee abstract state, and similarly extract the abstract and mostly-concrete heaps from the abstract exit state.

CONCERTO also provides an API for the AI to yield into the mostly-concrete interpreter when it encounters a call back into the framework. The AI calls this API method with a mostly-concrete receiver and arguments as well as the abstract caller state. The mostly-concrete interpreter extracts the two heaps from this caller state, binds the argument and receiver values, and begins executing the called method. When execution of the method completes, CONCERTO injects the resulting mostly-concrete and abstract heaps into the provided abstract caller state, and returns this updated state and a mostly-concrete return value to the AI.

\subsection{Context-Sensitivity and Mostly-Concrete Interpretation}

CONCERTO supports context-sensitive analyses. Our implementation is polymorphic over the type of contexts, leaving the representation entirely to the client abstract interpretation. When computing the context for an application method call, CONCERTO passes information about the current state, call site, and call stack to a mkContext method exposed by the AI. mkContext is responsible for computing the analysis context $C$ for the method call and returning it to CONCERTO, which passes the computed context to the interpret function as described above.

At application-to-framework calls, CONCERTO spawns a fresh mostly-concrete interpreter and runs it until the called method returns. Where possible, this interpreter unrolls all statically bounded loops and unfolds the call-graph (effectively giving unlimited context-sensitivity and some pathsensitivity). However, due to nondeterministic program inputs or imprecision in the abstract interpreter, mostly-concrete interpretation may encounter nondeterministic conditionals, loops, and unbounded recursive cycles.

To handle nondeterministic choice, CONCERTO could fork two interpreters and continue execution down each path in parallel as in Klee [Cadar et al. 2008] or Java PathFinder [Brat et al. 2000]. While sound, this approach would encounter the exponential explosion of paths common in symbolic execution. Instead, CONCERTo forks two interpreters at nondeterministic branches and executes 
both branches in parallel up to the conditional control-flow join point. At the control-flow join point, the two interpreter's states are joined and execution continues along a single thread of execution.

To ensure termination in the presence of nondeterministic loops or unbounded recursion, the mostly-concrete interpreter is instrumented to detect potentially infinite loop unrolling or call-graph unfolding and then falls back on over-approximation using widening.

\subsection{Allowing State Separation Violations}

The state separation hypothesis applies to the language presented thus far; the choice of whether a variable is modeled by an abstract value or mostly-concrete value could be made based on types, and operations on abstract values can only occur in the abstract interpreter and similarly for mostly-concrete values. However, the state separation hypothesis is violated if we add primitive types, interfaces, and common library types such as Hashtable to our supported subset of Java; e.g., frameworks may interrogate or modify an integer produced by application code. Although we could restrict the use of primitives and libraries to only application or framework code, and further require that all implementers of an interface must be either application or framework classes, such a restriction would be unrealistic. We therefore describe how to handle these features as well as direct mutation of application objects by the framework and vice versa.

8.4.1 Primitive Types and Operations. Our limited subset of Java supports only integers with basic arithmetic operations and comparisons. Integer values are represented in the mostly-concrete interpreter with a sum type: $\wp(\mathbb{N})^{\top}+\widehat{A}$. When an integer abstraction of type $\widehat{A}$ flows into a framework method we automatically lift it into the sum type. We use $\widehat{A}$ as the abstract representation of objects and integers: in practice we expect that internally $\widehat{A}$ is a union of objects and integers abstractions.

When executing an arithmetic or comparison operation, CONCERTO checks if both operands are of type $\wp(\mathbb{N})^{\top}$. If so, then the interpreter executes the concrete arithmetic operation lifted to the $\wp(\mathbb{N})^{\top}$ domain. If one or both of the operands are of type $\widehat{A}$, then Concerto uses a lift $: \wp(\mathbb{N})^{\top} \rightarrow \widehat{A}$ method exported by the abstract interpreter to convert the powerset representation into an $\widehat{A}$ representation. After conversion, CONCERTO calls methods exposed by the abstract interpretation that perform the primitive operations on elements of $\widehat{A}$. Finally, in cases such as array indexing, CONCERTo may need to transform $\wp(\mathbb{N})^{\top}+\widehat{A}$ into $\wp(\mathbb{N})^{\top}$. The abstract interpretation must also expose a function lower : $\widehat{A} \rightarrow \wp(\mathbb{N})^{\top}$. A sound choice for this function is to simply return $\top_{F}$.

At calls from the framework into the application, CONCERTO passes the sum representation directly to the abstract interpretation which may lift this sum type into a native representation. In practice, abstract interpretations gain precision by using the sum representation for integers, and lifting to a native representation on demand for arithmetic and comparison operations.

8.4.2 Interfaces and Library Types. An interface I may have implementers in the application and the framework. Thus, given a variable/field of type $I$, it may be unknown whether that variable/field contains an instance of a framework or application class. We resolve this ambiguity by requiring that the abstract interpretation and mostly-concrete interpreter use a combined object representation $\wp\left(V_{f}\right) \times \widehat{A}$ for values with an interface type. Intuitively, $\left\langle v_{f}, \widehat{a}\right\rangle: \wp\left(V_{f}\right)^{\top} \times \widehat{A}$ represents either a framework object that is abstracted by $v_{f}$ or an application object abstracted by $\widehat{a}$. In the case that one of the components is the least element in its respective lattice then the interpretation of combined value is the interpretation of the non-bottom component. In principle, we could have used this product representation for primitive types, but found that in practice the sum type representation is easier to use.

When the mostly-concrete interpreter encounters a method call on an interface, it splits the receiver into its two components, and then performs a concrete method call on the concrete component while simultaneously performing the abstract method call by yielding into the abstract 
interpreter. The results from both method calls are then merged using the appropriate join operations and execution continues. CONCERTO exports an API that performs the symmetric operation for interface calls encountered in application code by the abstract interpreter.

Both framework and application code may use the same standard libraries, breaking our simplifying assumption that the types used in the application and framework are disjoint. For example, Java types like ArrayList, HashMap, etc. are ubiquitous. CONCERTO supports these library types using the same product representation used for interfaces. Unlike interfaces, the choice of whether to model library objects using a mostly-concrete or abstract representation is not based on static type information, but on the allocation site. For example, an ArrayList allocated in the application will be modeled using abstract values, whereas an ArrayList allocated in the framework will be modeled mostly-concretely. This approach is possible because we assume that library types, like interfaces, only export object-oriented interfaces and CONCERTO does not need to support framework code that directly modifies the internal state of a library objects allocated in the application and vice versa.

8.4.3 Direct Field Access. We have so far assumed that the framework never directly accesses or mutates application object fields and vice-versa. As argued in Section 2, we expect this assumption holds for the vast majority framework-based applications. However, our approach can still be applied in the cases where the assumption does not hold, albeit with some precision penalty We describe how to support framework code that reads and writes application object fields; the approach for the application code directly accessing framework fields is symmetric.

We first consider the case where framework code reads an application object field. Recall that we model operations on application fields as aops and that we use an extremely coarse model for aops in our mostly-concrete semantics. Thus, we can soundly model reads of application object fields as simply returning the maximal element from the appropriate lattice. For example, the mostly-concrete interpreter may use $T_{\widehat{A}}$ to model the value read from a field of application type. Any future operations on this read value will necessarily be imprecise; the exact extent of this imprecision will depend on how the read value is used by the program.

Our approach for handling direct mutations of application fields is broadly similar. As field writes are modeled as aops that side effect the abstract heap, mutations are coarsely modeled by simply havocing the abstract heap, i.e. the interpretation of a field write returns $T_{\widehat{H_{a}}}$, where $\widehat{H_{a}}$ is the domain of abstract heaps. This coarse approach will cause greater imprecision compared to the field read case above, but we contend that direct field mutations are exceedingly rare in practice. As frameworks and applications are developed independently from one another, the framework implementation cannot guarantee any application-specific object invariants are preserved by a field mutation. A similar argument applies for direct mutations of framework field by the application code. Thus, we expect any imprecision introduced by this coarse modeling to be limited for real-world applications.

\section{EVALUATION}

To evaluate the feasibility and benefits of our combined analysis approach, we implemented a small "web application framework" called YAwn (Your Analysis' Worst Nightmare) in the subset of Java supported by our prototype implementation. YAwn implements an accept loop which parses requests received on our language's nondeterministic IO stream and routes these requests to application defined handlers. YAwn contains several difficult-to-analyze features found in realworld frameworks, including dependency injection, an embedded Lisp interpreter, and indirect flow. The dependency injection component and the Lisp interpreter heavily used reflection, and the run-time behavior of all three features is determined by the contents of a configuration file. 
Table 1. Summary of abstract interpretations. CS is the context-sensitivity of the analysis if applicable. PS indicates if the analysis is path-sensitive.

\begin{tabular}{|c|c|c|c|c|c|}
\hline Name & CS? & Heap & Domain & Relational? & PS? \\
\hline PTA & No & Type-based & Reaching Types & No & No \\
\hline IFLOW & Caller Method & Type-based & Access Paths/Reaching Types & No & No \\
\hline ABC & Call site 1-CFA & Abstract Location & Pentagons/Abstract Locations & Yes & Yes \\
\hline
\end{tabular}

Table 2. Number of reports issued and execution times of the interpreters with (Conc.) and without (Std. AI) Concerto. t/o indicates a timeout. For a discussion of the PTA results, see the main text.

\begin{tabular}{|c||c|c||c|c|}
\hline Analysis & Time (Conc.) & Time (Std. AI) & Reports (CoNC.) & Reports (Std. AI) \\
\hline PTA & $4.7 \mathrm{~s}$ & $1282.7 \mathrm{~s}$ & - & - \\
\hline ABC & $8.8 \mathrm{~s}$ & $\mathrm{t} / \mathrm{o}$ & 0 & 2 \\
\hline IFLOW & $4.6 \mathrm{~s}$ & $\mathrm{t} / \mathrm{o}$ & 3 & 7 \\
\hline
\end{tabular}

We implemented a simple application using the YAwn framework. The application's primary functionality is implemented as a collection of request handlers which perform simple mathematical operations (e.g., summing two integers) on request parameters. The application uses in-memory state and a simulated database layer implemented as standalone modules. These handlers and modules are constructed and wired together using YAwn's dependency injection mechanism. YAwN also includes a filtering mechanism to preprocess requests. Our application applies a filter that uses YAwN's embedded Lisp interpreter to run a filtering program specified in the application's configuration file.

Next, we implemented three abstract interpreters that use different abstract domains, heap representations, and context sensitivity. These analyses are summarized in Table 1. PTA performs VTA-style [Sundaresan et al. 2000] call-graph construction using a type-based heap where abstract addresses are sets of type names. IFLow is an information flow integrity analysis [Denning 1976] to find flows from untrusted sources to sensitive sinks. It uses the caller method as the context when analyzing a callee. For the heap abstraction, IFLow reuses the type-based heap from the PTA interpreter, and extends the reaching type domain with k-limited access paths [Deutsch 1994; Jones and Muchnick 1979] that track which heap locations are tainted. Finally, the most complex (and expensive) analysis is ABC, an array bounds checker. Aвс uses call site 1-CFA for contexts, and uses an abstract heap that maps abstract locations to abstract objects. An abstract location is pair consisting of an allocation site and the context in which the allocation occurred. Object values are abstract by powersets of abstract locations. Integers are abstracted with an approximation of the reduced product [Cousot and Cousot 1979b] of the Interval domain Intv and inequalities between access paths, giving a weakly relational Pentagon domain [Logozzo and Fähndrich 2008]. As the choice of $\widehat{A}$ must be non-relational, the abstract representation of integers in $\widehat{A}$ is simply Intv. ABC also propagates inequalities induced by comparison operators making it partially path sensitive.

Finally, we ran each interpreter over the application twice: once using CONCERTO and once with standard abstract interpretation. Each run had a one hour time budget, and we measured the total time of each run. After each run, we collected call-graph information (РTA) or any alarms reported (ABC \& IFLOW). In the event of a timeout, we collected any information computed up to that point.

For every analysis, CONCERTO vastly outperformed plain abstract interpretation as shown in Table 2. Under plain abstract interpretation, ABC and IFLOw timed out while PTA took approximately $275 \times$ longer than combined interpretation. The ABC and IFLOw timeouts were caused by enormous strongly connected components due to sound but imprecise modeling of YAwN's use of reflection and indirection. Even with widening, propagating information through these cycles overwhelmed 
the abstract interpreters. PTA also encountered large strongly connected components, but the lack of context-sensitivity and simplicity of the abstract domains mitigated the performance impacts.

Further, the quality of analysis results was significantly worse with plain interpretation compared to CONCERTO. To evaluate the precision of $\mathrm{ABC}$ and IFLOw, we classified the alarms reported as either true or false positives. Combined interpretation correctly found all 3 information leaks in our test application and also successfully verified that the application was free of out-of-bounds array accesses. In contrast, plain abstract interpretation reported 7 leaks and and 2 out-of-bounds accesses, respectively. For the array bounds checker, all these reports were false positives, and all but 3 were false positives for the information flow analysis. Additionally, as these results were collected after timeouts, they represent a lower bound on the imprecision of ABC and IFLow.

PTA does not find bugs, but produces a call-graph for a downstream analysis; we include it in our experiments to demonstrate the impact of combined interpretation on resolving reflective invocations in framework code. As a representative example, under plain interpretation PTA resolved the reflective allocations in the dependency injection facility to 38 possible types, compared to just 15 types under combined interpretation. Similarly, within the Lisp interpreter, plain interpretation resolved the reflective invocations to as many as 30 and no fewer than 8 callees, whereas combined interpretation resolved every invocation to a single callee.

\section{RELATED WORK}

There has been considerable work on improving analysis precision for framework-based applications by using the information in configuration files, particularly for Android applications [Arzt et al. 2014; Blackshear et al. 2015]. The Frameworks for Frameworks system by Sridharan et al. [2011] generalizes these efforts, providing a framework for writing framework models. Unfortunately, manually providing a framework model is an enormous effort that is rarely reusable across different frameworks. In contrast, once the concrete semantics for a language are specified, combined interpretation can in principle be used with any framework without further effort.

Several static analyses have resolved calls that use reflection [Barros et al. 2015; Li et al. 2015; Smaragdakis et al. 2015]. To succeed, these techniques rely on programs using reflection APIs with constant strings and type-casting the results in stylized ways. These patterns rarely occur in framework implementations. In addition, these techniques are tailored to specific APIs (i.e., Java's reflection API) and adapting them to other domains requires non-trivial work by the analysis designer. Moreover, frameworks are difficult to analyze for reasons beyond their use of reflection.

Many efforts have combined dynamic and static analysis, yielding "blended" or "hybrid" analyses. Some hybrid analyses use information recorded during dynamic executions of the program to improve the static-analysis precision [Csallner et al. 2008; Dufour et al. 2007; Grech et al. 2017; Ren and Foster 2016; Wei and Ryder 2013]. For example, TamiFlex by Bodden et al. [2011] instruments an application as it runs a representative workload to record the callees of reflective calls. These approaches generally suffer from unsoundness; even representative workloads rarely exercise all possible execution paths. Symmetrically, other researchers have run a dynamic analysis seeded with information produced by a static analysis [Balzarotti et al. 2008; Chugh et al. 2009; Lam et al. 2008]. As with the above approaches, any dynamic analysis will almost certainly be unsound.

Other researchers have explored other approaches to combining analyses. For example, [Fink et al. 2008] runs a series of increasingly precise analyses to prune false positives left by earlier analyses. Work on tunable analyses of JavaScript [Ko et al. 2015] uses the results of a pre-analysis to restrict the abstract states explored by an abstract interpretation. [Ferrara 2014] explores combining a value and heap analysis to improve precision in abstract interpretation. Their approach embeds information produced by the heap analysis into the domain of the value analysis. This embedding closely mirrors how CONCERTO embeds abstract values into the mostly-concrete state and vice versa. Other

Proc. ACM Program. Lang., Vol. 3, No. POPL, Article 43. Publication date: January 2019. 
researchers have combined different execution strategies for different portions of code [Avgerinos et al. 2014; Chipounov et al. 2011]. For example, [Chipounov et al. 2011] switch to fully concrete execution on portions of the system under test. Their approach requires concretizing symbolic values and then checking that doing so does not prune any feasible paths. Concretization is performed lazily, which parallels how CONCERTO threads abstract values through mostly-concrete interpretation. However, due to the state separation hypothesis, CONCERTO can avoid all concretization.

Our combined interpretation bears similarity to concolic testing [Godefroid et al. 2005; Sen and Agha 2006; Sen et al. 2005]. Concolic testing performs symbolic and concrete execution in parallel, but falls back on concrete values in the symbolic interpreter when it encounters an expression outside of the logic of the underlying theorem prover. This approach is similar to how ConCERTO uses concrete execution to precisely reason about difficult-to-analyze code. Similarly, as noted in Section 2, our technique is similar to partial evaluation [Futamura 1999; Mogensen 1995]. However, partial evaluation is typically used for optimization [Brown and Palsberg 2017; Jones et al. 1993], and the full resolution of reflection is usually an orthogonal concern. We are unaware of work trying to use partial evaluation to handle difficult-to-analyze framework code for sound program analysis, and we believe CONCERTO is less brittle than a partial evaluation approach to this problem.

Finally, many researchers have improved analysis precision by combining abstract domains [Brauer et al. 2010; Cousot et al. 2006; Fähndrich and Logozzo 2010; Ferrara 2010; Laviron and Logozzo 2009; Logozzo and Fähndrich 2008; Toubhans et al. 2013; Zanioli et al. 2012], via the reduced product [Cousot and Cousot 1979b], reduced tensor product [Nielson 1985], etc. Astree [Cousot et al. 2005] in particular is an industry tool that computes an approximate reduced product by propagating information through a tree of abstract domains [Cousot et al. 2006]. Our approach could be formalized as a degenerate case of this framework, where the exchange of information between the abstract and mostly-concrete interpreters takes place at the transition points via the communication channels described in [Cousot et al. 2006]. However, while the reduced product domain found in Astree and other abstract interpretations typically exchange information about the same program point between multiple domains, under CONCERTO each program statement is analyzed by either mostly-concrete or abstract interpretation. Further, our subfixpoint iteration strategy is significantly different from the one described in [Cousot et al. 2006].

\section{CONCLUSIONS AND FUTURE WORK}

We presented CONCERTO, a framework for soundly combining concrete and abstract interpretation. CONCERTO targets framework-based applications that use difficult-to-analyze reflection, metaprogramming, and abstractions. This combination is possible because framework-based applications in practice satisfy a state separation hypothesis which CONCERTo exploits to opaquely embed abstract values into a mostly-concrete interpreter. Our combination supports any abstract interpreter that satisfies a modest set of conditions, and yields significant improvements in initial experiments with a research prototype. Further generalizing our framework, in particular supporting alternative definitions of soundness (e.g., via concretization function [Cousot and Cousot 1992b]) is one area of future work. We also plan to extend our initial research prototype to support the full Java language and real-world frameworks.

\section{ACKNOWLEDGMENTS}

We thank James Wilcox, Ben Hardekopf, Bill Harris, James Bornholt, and Jared Roesch for their comments on early drafts of this work. Thanks also to Pavel Panchekha for his insight on our proofs. Finally, we thank the anonymous reviewers, in particular our shepherd, for their comments and feedback on this work. This material is based upon work supported in part by DARPA under agreement number FA8750-16-2-0032. 


\section{REFERENCES}

Steven Arzt, Siegfried Rasthofer, Christian Fritz, Eric Bodden, Alexandre Bartel, Jacques Klein, Yves Le Traon, Damien Octeau, and Patrick McDaniel. 2014. FlowDroid: Precise Context, Flow, Field, Object-sensitive and Lifecycle-aware Taint Analysis for Android Apps. In PLDI.

Thanassis Avgerinos, Alexandre Rebert, Sang Kil Cha, and David Brumley. 2014. Enhancing symbolic execution with veritesting. In ICSE.

Davide Balzarotti, Marco Cova, Vika Felmetsger, Nenad Jovanovic, Engin Kirda, Christopher Kruegel, and Giovanni Vigna 2008. Saner: Composing static and dynamic analysis to validate sanitization in web applications. In Symposium on Security and Privacy.

Paulo Barros, René Just, Suzanne Millstein, Paul Vines, Werner Dietl, Michael D Ernst, et al. 2015. Static analysis of implicit control flow: Resolving Java reflection and Android intents. In ASE.

Sam Blackshear, Alexandra Gendreau, and Bor-Yuh Evan Chang. 2015. Droidel: A general approach to android framework modeling. In SOAP.

Eric Bodden, Andreas Sewe, Jan Sinschek, Hela Oueslati, and Mira Mezini. 2011. Taming reflection: Aiding static analysis in the presence of reflection and custom class loaders. In ICSE.

François Bourdoncle. 1993. Efficient chaotic iteration strategies with widenings. In Formal Methods in Programming and their Applications.

Guillaume Brat, Klaus Havelund, SeungJoon Park, and Willem Visser. 2000. Java PathFinder-second generation of a Java model checker. In Workshop on Advances in Verification.

Jörg Brauer, Thomas Noll, and Bastian Schlich. 2010. Interval analysis of microcontroller code using abstract interpretation of hardware and software. In Workshop on Software \& Compilers for Embedded Systems.

Matt Brown and Jens Palsberg. 2017. Jones-optimal partial evaluation by specialization-safe normalization. Proc. ACM Program. Lang. 2, POPL (2017), 14.

Cristian Cadar, Daniel Dunbar, Dawson R Engler, et al. 2008. KLEE: Unassisted and Automatic Generation of High-Coverage Tests for Complex Systems Programs. In OSDI.

Vitaly Chipounov, Volodymyr Kuznetsov, and George Candea. 2011. S2E: A Platform for In-vivo Multi-path Analysis of Software Systems. In ASPLOS.

Ravi Chugh, Jeffrey A. Meister, Ranjit Jhala, and Sorin Lerner. 2009. Staged Information Flow for Javascript. In PLDI.

Patrick Cousot. 1977. Asynchronous iterative methods for solving a fixed point system of monotone equations in a complete lattice. Res. rep. RR 88 (1977).

Patrick Cousot and Radhia Cousot. 1977. Abstract interpretation: a unified lattice model for static analysis of programs by construction or approximation of fixpoints. In POPL.

Patrick Cousot and Radhia Cousot. 1979a. Constructive versions of Tarski's fixed point theorems. Pacific journal of Mathematics 82, 1 (1979).

Patrick Cousot and Radhia Cousot. 1979b. Systematic design of program analysis frameworks. In POPL. ACM.

Patrick Cousot and Radhia Cousot. 1992a. Abstract interpretation and application to logic programs. The fournal of Logic Programming 13, 2-3 (1992), 103-179.

Patrick Cousot and Radhia Cousot. 1992b. Abstract interpretation frameworks. Journal of logic and computation 2, 4 (1992).

Patrick Cousot, Radhia Cousot, Jérôme Feret, Laurent Mauborgne, Antoine Miné, David Monniaux, and Xavier Rival. 2005 The ASTRÉE analyzer. In ESOP.

Patrick Cousot, Radhia Cousot, Jérôme Feret, Laurent Mauborgne, Antoine Miné, David Monniaux, and Xavier Rival. 2006 Combination of abstractions in the ASTRÉE static analyzer. In Annual Asian Computing Science Conference.

Christoph Csallner, Yannis Smaragdakis, and Tao Xie. 2008. DSD-Crasher: A hybrid analysis tool for bug finding. TOSEM 17, 2 (2008), 8.

Dorothy E Denning. 1976. A lattice model of secure information flow. Commun. ACM 19, 5 (1976)

Alain Deutsch. 1994. Interprocedural May-alias Analysis for Pointers: Beyond K-limiting. In PLDI.

Bruno Dufour, Barbara G. Ryder, and Gary Sevitsky. 2007. Blended analysis for performance understanding of frameworkbased applications. In ISSTA.

Manuel Fähndrich and Francesco Logozzo. 2010. Static contract checking with abstract interpretation. In Formal Verification of Object-Oriented Software.

Pietro Ferrara. 2010. Static type analysis of pattern matching by abstract interpretation. In Formal Techniques for Distributed Systems. Springer, 186-200.

Pietro Ferrara. 2014. Generic combination of heap and value analyses in abstract interpretation. In VMCAI.

Stephen J. Fink, Eran Yahav, Nurit Dor, G. Ramalingam, and Emmanuel Geay. 2008. Effective typestate verification in the presence of aliasing. TOSEM 17, 2 (2008).

Martin Fowler. 2004. Inversion of control containers and the dependency injection pattern. (2004). 
Yoshihiko Futamura. 1999. Partial evaluation of computation process-an approach to a compiler-compiler. Higher-Order and Symbolic Computation 12, 4 (1999), 381-391.

Patrice Godefroid, Nils Klarlund, and Koushik Sen. 2005. DART: Directed Automated Random Testing. In PLDI.

Neville Grech, George Fourtounis, Adrian Francalanza, and Yannis Smaragdakis. 2017. Heaps Don't Lie: Countering Unsoundness with Heap Snapshots. Proc. ACM Program. Lang. 1, OOPSLA, Article 68 (2017).

Neil D Jones, Carsten K Gomard, and Peter Sestoft. 1993. Partial evaluation and automatic program generation. Peter Sestoft.

Neil D Jones and Steven S Muchnick. 1979. Flow analysis and optimization of LISP-like structures. In POPL.

John B Kam and Jeffrey D Ullman. 1977. Monotone data flow analysis frameworks. Acta Informatica 7, 3 (1977), $305-317$.

Yoonseok Ko, Hongki Lee, Julian Dolby, and Sukyoung Ryu. 2015. Practically Tunable Static Analysis Framework for LargeScale JavaScript Applications. In ASE.

Monica S. Lam, Michael Martin, Benjamin Livshits, and John Whaley. 2008. Securing web applications with static and dynamic information flow tracking. In Partial Evaluation and Semantics-based Program Manipulation.

Vincent Laviron and Francesco Logozzo. 2009. Subpolyhedra: A (more) scalable approach to infer linear inequalities. In VMCAI.

Yue Li, Tian Tan, and Jingling Xue. 2015. Effective soundness-guided reflection analysis. In SAS.

Francesco Logozzo and Manuel Fähndrich. 2008. Pentagons: a weakly relational abstract domain for the efficient validation of array accesses. In Symposium on Applied Computing.

Torben Mogensen. 1995. Self-applicable online partial evaluation of the pure lambda calculus. In Symposium on Partial Evaluation and Semantics-based Program Manipulation.

Flemming Nielson. 1985. Tensor products generalize the relational data flow analysis method. In 4th Hungarian Computer Science Conference. 211-225.

Brianna M. Ren and Jeffrey S. Foster. 2016. Just-in-time Static Type Checking for Dynamic Languages. In PLDI.

Koushik Sen and Gul Agha. 2006. CUTE and jCUTE: Concolic unit testing and explicit path model-checking tools. In CAV.

Koushik Sen, Darko Marinov, and Gul Agha. 2005. CUTE: A Concolic Unit Testing Engine for C. In ESEC/FSE.

Yannis Smaragdakis, George Balatsouras, George Kastrinis, and Martin Bravenboer. 2015. More sound static handling of Java reflection. In ASPLAS.

Manu Sridharan, Shay Artzi, Marco Pistoia, Salvatore Guarnieri, Omer Tripp, and Ryan Berg. 2011. F4F: Taint Analysis of Framework-based Web Applications. In OOPSLA.

Gregory T Sullivan. 2001. Dynamic partial evaluation. In Programs as Data Objects. 238-256.

Vijay Sundaresan, Laurie Hendren, Chrislain Razafimahefa, Raja Vallée-Rai, Patrick Lam, Etienne Gagnon, and Charles Godin. 2000. Practical Virtual Method Call Resolution for Java. In OOPSLA.

Alfred Tarski. 1955. A lattice-theoretical fixpoint theorem and its applications. Pacific journal of Mathematics 5, 2 (1955).

Antoine Toubhans, Bor-Yuh Evan Chang, and Xavier Rival. 2013. Reduced product combination of abstract domains for shapes. In VMCAI.

Shiyi Wei and Barbara G Ryder. 2013. Practical blended taint analysis for JavaScript. In ISSTA.

Matteo Zanioli, Pietro Ferrara, and Agostino Cortesi. 2012. SAILS: Static Analysis of Information Leakage with Sample. In Symposium on Applied Computing. 